\title{
Automated Labeling of Chest X-ray Images using a Quantitative Explainable Atlas-Based AI Model
}

\section{Doyun Kim}

Massachusetts General Hospital

Joowon Chung

https://orcid.org/0000-0003-1572-8056

Jongmun Choi

Massachusetts General Hospital

Marc Succi

Massachusetts General Hospital

John Conklin

Massachusetts General Hospital

Maria Gabriela Figueiro Longo

Massachusetts General Hospital

Jeanne Ackman

Massachusetts General Hospital https://orcid.org/0000-0001-5022-9530

\section{Brent Little}

Massachusetts General Hospital

Milena Petranovic

Massachusetts General Hospital

\section{Mannudeep Kalra}

Massachusetts General Hospital https://orcid.org/0000-0001-9938-7476

\section{Michael Lev}

Massachusetts General Hospital https://orcid.org/0000-0003-0236-7319

Synho Do ( $\nabla$ sdo@mgh.harvard.edu )

Massachusetts General Hospital https://orcid.org/0000-0001-6211-7050

\section{Article}

Keywords: labeling, chest x-ray images, open-access medical imaging datasets, Al models

Posted Date: August 23rd, 2021

DOI: https://doi.org/10.21203/rs.3.rs-830690/v1 
License: (c) (i) This work is licensed under a Creative Commons Attribution 4.0 International License. Read Full License

Version of Record: A version of this preprint was published at Nature Communications on April 6th, 2022. See the published version at https://doi.org/10.1038/s41467-022-29437-8. 
2 Automated Labeling of Chest X-ray Images using a Quantitative Explainable Atlas-Based AI Model

12 The inability to accurately, efficiently label large, open-access medical imaging datasets limits the 13 widespread implementation of artificial intelligence models in healthcare. There have been few 14 attempts, however, to automate the annotation of such public databases; one approach, for 15 example, focused on labor-intensive, manual labeling of subsets of these datasets to be used to 16 train new models. In this study, we describe a method for standardized, automated labeling based 17 on similarity to a previously validated, explainable Al model (XAI), using an atlas-based approach, 18 for which the user can specify a quantitative threshold for a desired level of accuracy, the 19 "probability-of-similarity" (pSim) metric. We showed that our xAl model, by calculating the pSim 20 values for each feature based on comparison to its training-set derived reference atlas, could 21 automatically label the external datasets to a user-selected, high level of accuracy, equaling or 22 exceeding that of human experts. 


\section{Introduction}

25 The implementation of medical artificial intelligence (AI) into clinical practice in general, and 26 radiology practice in particular, has in large part been limited by the time, cost, and expertise 27 required to accurately label very large imaging datasets, which can serve as "platinum level" 28 ground truth for training clinically relevant Al models. The ability to automatically and efficiently 29 annotate large external datasets, to a user-selected level-of-accuracy, may therefore be of 30 considerable value in developing impactful, important, medical Al models that bring added value 31 to, and are widely accepted by, the healthcare community. Such an approach not only has the 32 potential to benefit re-training to improve the accuracy of existing Al models, but also - through 33 using explainable, atlas-based methodology [1] - may help to standardize labeling of open-source 34 datasets [2-5], for which the provided labels can be noisy, inaccurate, or absent. Such 35 standardization may, in turn, reduce the number of datapoints required for accurate model 36 building, facilitating training and re-training from initial small but well annotated datasets [1, 6].

In this study, we develop and demonstrate a method for standardized, automated labeling based on similarity to a previously validated explainable AI model (xAI), using an atlas-based approach

40 for which the user can specify a quantitative threshold for a desired level of accuracy (the 41 "probability-of-similarity", or "pSim" metric). The pSim values range from a "baseline" likelihood of 42 similarity ( $p$ Sim $=0$, least selective) to a "maximal" likelihood of similarity ( $p$ Sim=1, most selective);

43 pSim is computed by comparison between test-set derived image features and image features 44 "remembered"/retrieved from the model's reference atlas (i.e., "library"). This atlas is constructed 45 during model building (Fig. 1a) from the training set cases (Fig. 1b). The calculated pSim value 46 reflects the harmonic mean between two model related parameters, the "patch similarity" and the 47 "confidence" (Methods, Figs. 1b,c). 
49 Specifically, we applied our existing Al model for detection of five different chest X-ray (CXR)

50 imaging features (cardiomegaly, atelectasis, pulmonary edema, pneumonia, and pleural effusion),

51 to three large open-source datasets - CheXpert [2], MIMIC [3], and NIH [4] - and compared the

52 resulting labels to those of 7 human expert radiologists. Of note, there is an inverse relationship

53 between the selected pSim threshold values and the number of cases identified (i.e., "captured")

54 by the model from the external dataset; in other words, the higher the threshold for likelihood of

55 similarity, the fewer cases that will be identified from the external database as "similar" to the

56 model labeled cases.

57

58 We showed that our XAI model, by calculating the pSim values for each feature based on 59 comparison to the model's training-set derived reference atlas, could automatically label the

60 external datasets to a user-selected, arbitrarily high level of accuracy, equaling or exceeding that

61 of human experts. Although the pSim threshold value required to achieve "maximal" similarity

62 varies by feature, once that value is identified - based on comparison of model labels to expert-

63 labeled ground truth - it can then be applied to the remaining external dataset, to identify cases

64 likely to be positive for that feature at a pre-determined, high confidence level. 


\section{Results}
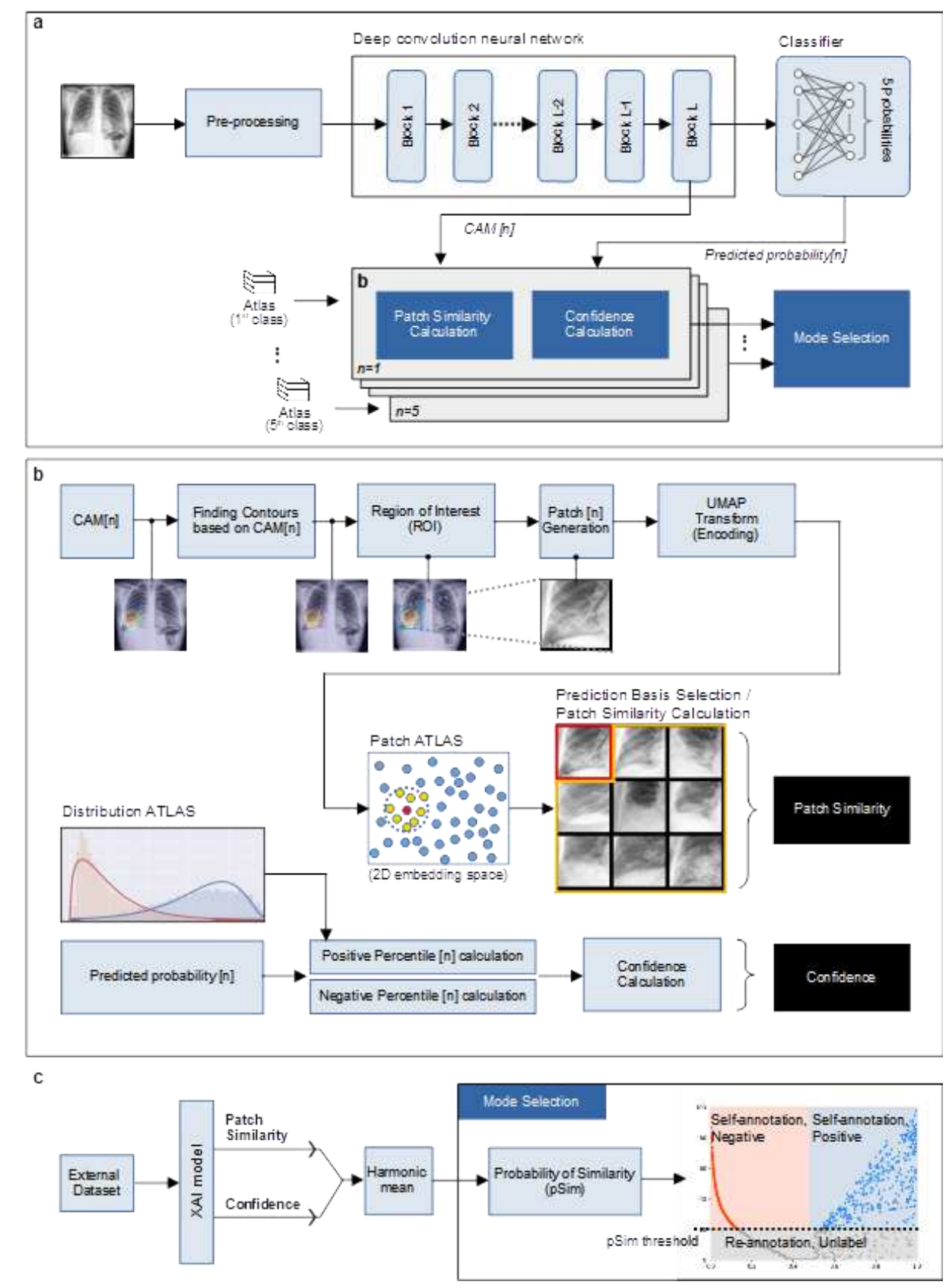

68 Fig. 1 | System overview. Standardized, automated labeling method, based on similarity to a 69 previously validated five-feature CXR detection xAl model, using an explainable atlas-based 70 approach. a, b The XAI model calculates "patch similarity" and "confidence" probabilities, based 71 on class activation mapping (CAM) [7, 8] and predicted probability from the model, for each 72 feature. c, The harmonic mean between the patch similarity and confidence xAl model outputs 73 are then used to calculate a "probability of similarity" (pSim) for each feature. 
75 System design. We developed an XAI model for detection of the following five different features 76 on posterior-anterior (PA) projection CXRs: cardiomegaly, atelectasis, pulmonary edema, 77 pneumonia, and pleural effusion (see Methods). As per previous reports, our model featured atlas 78 creation and prediction-basis calculation modules, for explainability (Fig. 1) [1]. The prediction79 basis was used to calculate a "patch similarity" value (a probability between 0 and 1). Our model 80 also included a "confidence" probability calculation module (Figs. 1a,b). The harmonic mean

81 between the patch similarity and confidence model outputs were used to calculate a quantitative

82 "probability of similarity" (pSim) value, between 0 and 1, for each feature studied (Fig. 1c).

84 xAl model development. CXR examinations performed at our institution from February 2015 85 through February 2019 were identified from our RIS (Radiology Information System) and PACS 86 (Picture Archiving and Communication System), resulting in a dataset of 440,852 studies.

87 Examinations were excluded if there was no associated radiology report, no view position 88 information (e.g. anteroposterior projection, "portable", etc.), or no essential patient identifiers 89 (including but not limited to medical record number, age, or gender). A total of 400,886 CXR 90 images from 267,180 examinations, representing 117,195 patients, together with their 91 corresponding radiology reports, were collected retrospectively (Supplementary Figure 1). Using 92 a rule-based Natural Language Processing (NLP) model (Supplementary Table 1), we 93 automatically extracted 20 pathological features from the radiology reports, which were assigned 94 one of the following three labels: "positive", "negative", or "ignore". After automated NLP data 95 mining and clean-up, we archived 151,700 anteroposterior (AP) CXR views from 49,096 patients 96 (58\% male, mean age 62 \pm 18 years) and 90,023 posteroanterior (PA) CXR views from 69,404 97 patients (50\% male, mean age $57 \pm 19$ years). We randomly selected 1000 images for each view 98 position as a test set; the remaining examinations, from non-overlapping patients, were separated 99 into training and validation sets (Supplementary Fig. 1). The labels for the training and validation 
sets were determined exclusively from the automated NLP assignments, whereas those for the

101 test set were determined by consensus of three U.S. board-certified radiologists at our institution

102 (further details provided in Supplementary Table 1), using the "Mark-it" tool

103 (https://markit.mgh.harvard.edu, MA, USA) for annotation [9]. This xAl model achieved a mean

104 Area Under the Receiver Operating Characteristic (AUROC) curve [10] of $0.95 \pm 0.02$ for detection

105 of the five features (Supplementary Table 2) in our initial, independent test set (Methods).

Auto-labeling model performance applied to three open-source datasets. We applied our XAI CXR auto-labeling model to the available PA CXR images from three large open-source datasets; CheXpert ( $n=29,420$ PA CXR's), MIMIC $(n=71,223)$, and NIH $(n=67,310)$ [2-4]. To assess labeling accuracy, we randomly selected a subset of "positive" and "negative" cases as determined by the model for each of the five features, distributed equally in each of ten pSim value ranges $(0-0.1,0.1-0.2,0.2-0.3, \ldots, 0.9-1.0)$, for expert review (Fig. 2). Ground truth (GT) was defined as the majority consensus of 7 expert sub-specialist radiologists (three with 12-25

114 years' experience in thoracic radiology and four with 1-6 years' experience in emergency radiology); GT and individual ratings of each reader, for each feature (cardiomegaly, atelectasis,

116 pulmonary edema, pneumonia, and pleural effusion), in each of the pSim value ranges, are shown 117 in panel 1 of Fig. 2 (upper left). In Fig. 2 panel 2 (upper right), we graph the relationship between 118 the pSim value applied for the model's auto-labeling (x-axis) and both the (i) "positive predictive 119 value" and "negative predictive value" of the model's ratings, versus ground truth; and the (ii) 120 model's "true positive capture rate" and "true negative capture rate", defined respectively as the 121 total true positive (by GT) divided by the total positive (by GT), and the total true negative (by GT) 122 divided by the total negative (by GT). In panels 3 (lower left) and 4 (lower right), respectively, the 123 number of false positive (by GT) and false negative (by GT) cases rated by the model at each 124 pSim threshold value (x-axis), are shown, stratified by dataset (CheXpert, MIMIC, or NIH), with 125 the "optimal", lowest pSim threshold achieving 100\% PPV or NPV, indicated. Of note, the lowest 
possible pSim threshold required for $100 \%$ PPV or NPV, corresponds to the maximal "correct

127 capture rate", as shown in panel 2.

128

129 Also, as shown in the text boxes in Fig. 2 panels 3 and 4, as well as in Fig. 3, model accuracy

130 compared favorably to that of the available pooled public labels of the external, open-source

131 datasets. Figure 3 additionally shows that the automated-labeling model's AUROC performance,

132 compared favorably to that of the individual expert radiologists, for each feature, at both the

133 pSim=0 "baseline" value labeling threshold and the "optimal" pSim value labeling threshold (i.e.,

134 the lowest pSim value achieving 100\% accuracy, as per Fig. 2 panels 3 and 4).

135

136 Sample auto-labeled CXR images that had complete agreement between all 7 expert radiogists 137 and the XAI model, positive for each of the five features studied, are shown in Fig. 4. The pSim 138 threshold values applied by the model for each image and the number/percent of PA CXR examinations with total agreement for each feature, are also shown. Of note, there were only 14 positive examinations identified by the model as "pneumonia" that had full agreement with each reader, of 50 total examinations labeled as positive for pneumonia (28\%). The percent positive labels with complete agreement for the other four features, as shown in the figure, were cardiomegaly $78 \%(39 / 50)$, atelectasis $46 \%$ (23/50), pulmonary edema $43 \%$ (17/40), and pleural 144 effusion $78 \%(39 / 50)$.

146 In Table 1, we applied our automated-labeling model to the three complete public, open source 147 CXR datasets: CheXpert $(n=29,420)$, MIMIC $(n=71,223)$, and $\mathrm{NIH}(n=67,310)$; in order to 148 demonstrate the magnitude of the number of cases "captured", at the optimized pSim threshold 149 value for maximal accuracy for each feature (PPV, NPV = 1; as per Fig. 2). Pooling the model's 150 labels for the three full public datasets (Table 1, far right) resulted in a "capture rate" of $80 \%$ for 151 cardiomegaly $(134,076 / 167,953), 28 \%$ for atelectasis $(47,436 / 167,953), 27 \%$ for pulmonary 

edema $(45,660 / 167,953), 20 \%$ for pneumonia $(33,308 / 167,953)$, and $68 \%$ for pleural effusion

$153(114,230 / 167,953)$. It is noteworthy that the model's mean CXR "capture rates" for the pooled

154 results from the three public datasets, closely corresponded to those shown in the graphs of Fig. 2.2 (a-e), for the subset of examinations $(n=90-100)$ labeled by both the model and the expert 156 radiologists.

Summary comparison of labeling efficiency, confidence metrics for the 5 auto-labeled features. For each of the five auto-labeled features (Fig. 5), we compared: (i) the percent of positively auto-labeled CXR's "captured" from the three pooled, full public datasets (from Table

161 1); (ii) the percent of cases with complete agreement between the model and all 7 expert readers 162 (from Fig. 4); (iii) the lowest pSim value such that PPV=1 (graphed as "1-pSim@PPV1"; from Fig. 2 panel 3), and (iv) the lowest pSim value such that NPV=1 (graphed as "1-pSim@NPV1"; from

164 Fig. 2 panel 4). Features with higher values of these parameters (e.g. cardiomegaly, pleural 165 effusion) corresponded to greater model auto-labeling efficiency and confidence; features with lower values (e.g. pneumonia, pulmonary edema) corresponded to lesser model auto-labeling 167 efficiency and confidence. Of note, for atelectasis, "1-pSim@PPV1" was higher than "1168 pSim@NPV1", indicating greater confidence that the model is correct in "ruling-in" this feature (i.e. 169 correctly auto-labeling true-positives) than in "ruling-out" this feature (i.e. correctly auto-labeling 170 true-negatives). This relationship was reversed for the other four features (e.g. greater confidence 171 that the model can correctly "rule-out" than "rule-in" pneumonia or pulmonary edema).

173 The pairwise kappa statistics estimating inter-observer variability among the 7 expert radiologists 174 are shown in Fig. 6, for each of the five auto-labeled features. The ranges for these values are as 175 follows: cardiomegaly $0.82-0.92$, pleural effusion $0.78-0.94$, atelectasis $0.47-0.78$, pulmonary 176 edema $0.57-0.86$, and pneumonia $0.38-0.80$. The distribution of these ranges correlates well with 177 the model's per feature auto-labeling efficiency and confidence metrics, shown in Fig. 5, with 
178 cardiomegaly and pleural effusion showing the most inter-rater agreement, and pneumonia, 179 pulmonary edema, and atelectasis showing the least. 
a. Cardiomegaly (total $n=100$ )
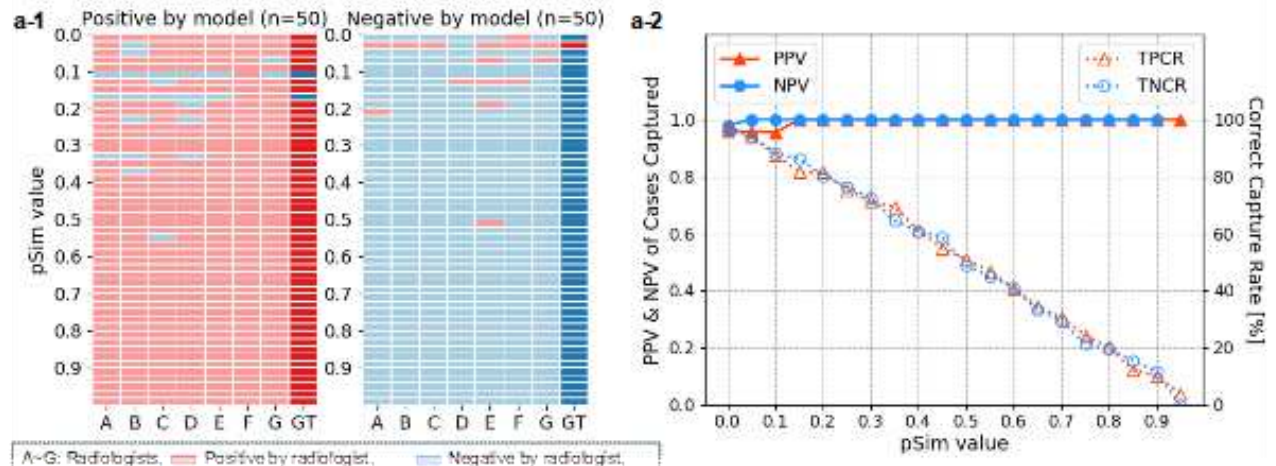

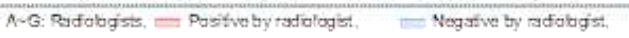



\section{a-3 EPby modelbased on Ground Truth (GT)}

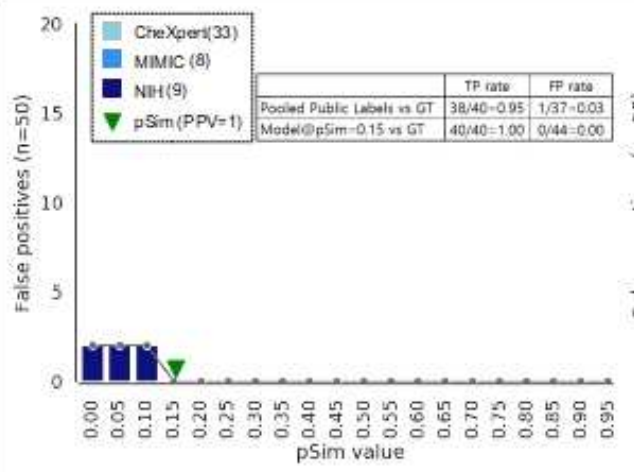

\section{a4 FNbymodel based on Ground Truth (GT)}

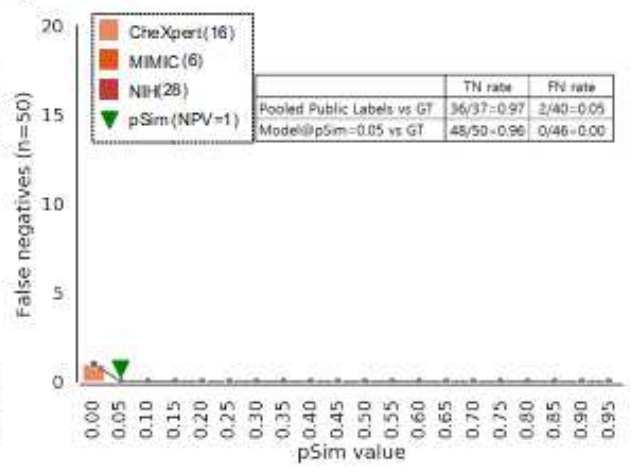

b. Atelectasis (total $n=100$ )


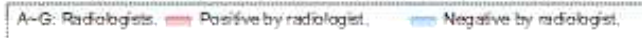

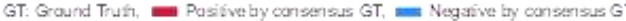


b-4 FN by model based on Ground Truth (GT)

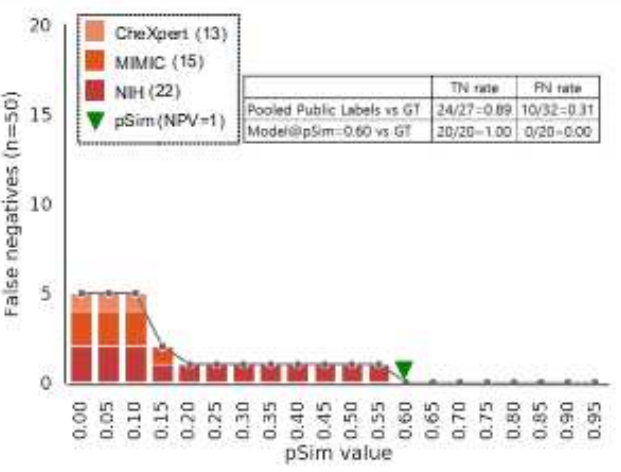


c. Pulmonary Edema (total $n=90$ )
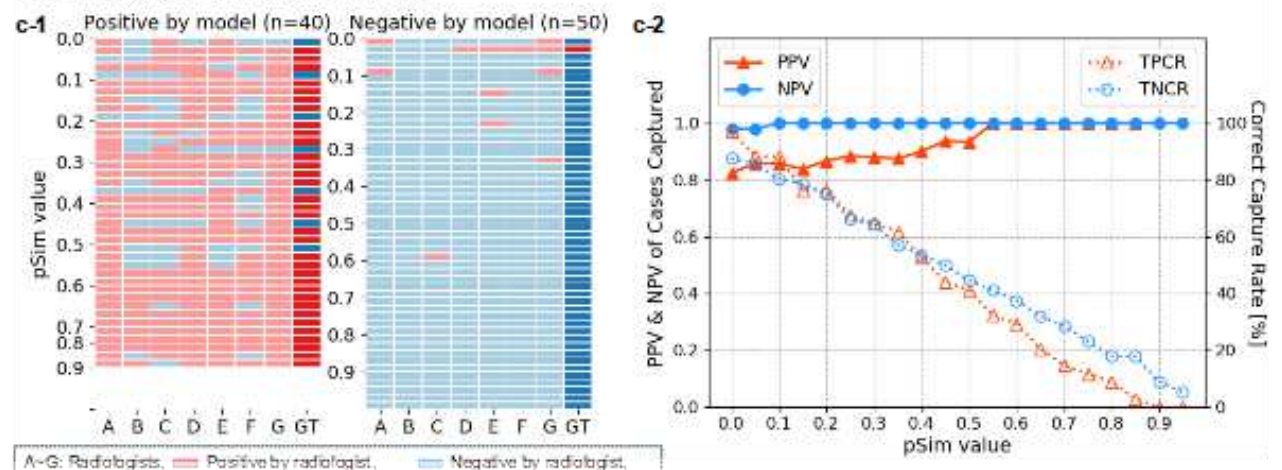

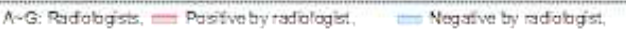

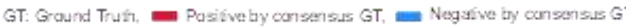

\section{C-3 FP by model based on Ground Truth (GT)}



\section{C-4 FN by model based on Ground Truth (GT)}

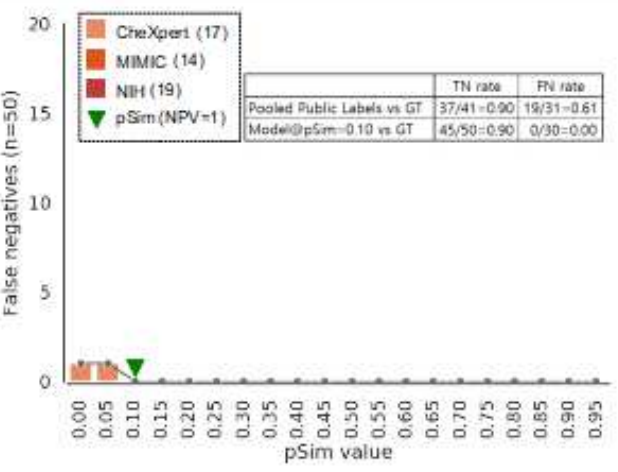

d. Pneumonia (total $n=100$ )




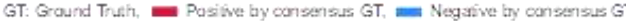

\section{d-3 FPby model based on Ground Truth (GT)}

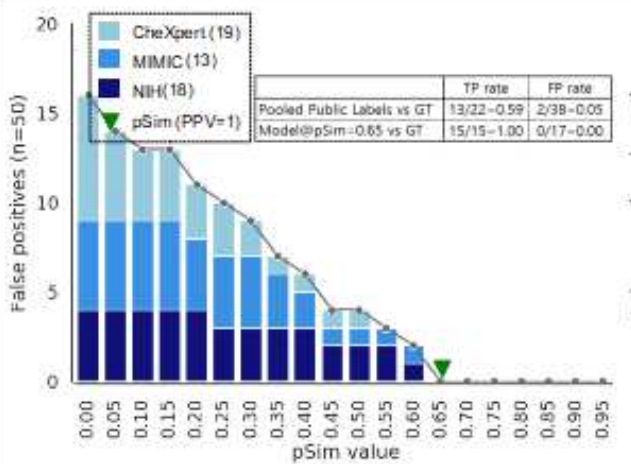

d-4 FN by model based on Ground Truth (GT)

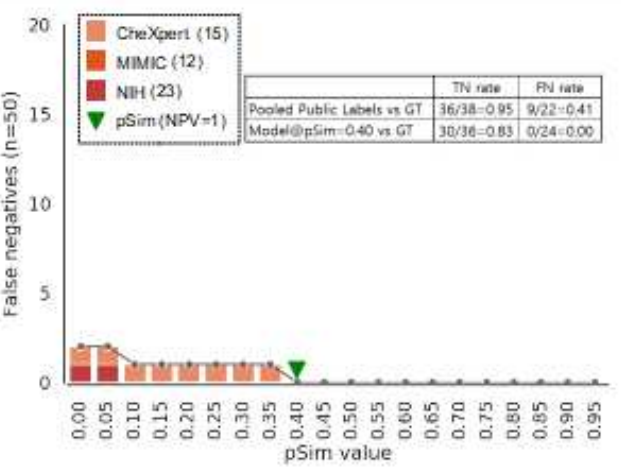




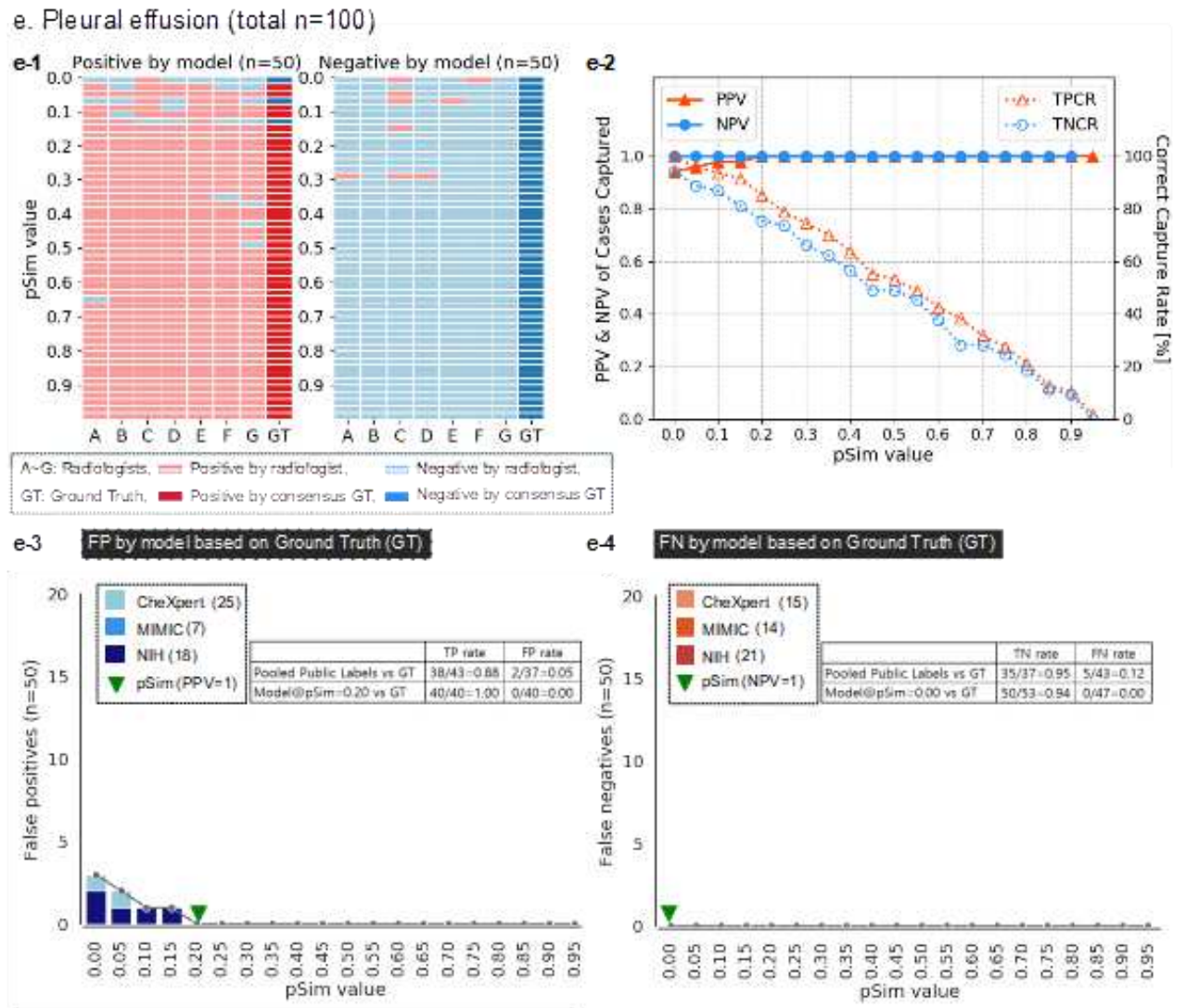

Fig. 2 | Automated-labeling model performance applied to three open-source CXR datasets, compared to consensus ground truth of 7 expert radiologists. We applied our XAI CXR autolabeling model to three large open-source datasets; CheXpert, MIMIC, and NIH. For each of the five features - a cardiomegaly, $\mathbf{b}$ atelectasis, $\mathbf{c}$ pulmonary edema, $\mathbf{d}$ pneumonia, and e pleural effusion - we randomly selected a subset of "positive" and "negative" cases as determined by the model, distributed equally in each of ten $p S i m$ value ranges $(0-0.1,0.1-0.2,0.2-0.3, \ldots, 0.9-1.0)$, 192 for expert review. In panel 1 (upper left), the positive (light red) and negative (light blue) ratings for each of the 7 individual readers (columns A-G) are displayed graphically, with the consensus ground truth (GT, determined by majority) shown in the last column (bold red or bold blue). In panel 2 (upper right), the "positive predictive values" (PPV=[true positive by GT]/[total positive by model], solid red triangles, $y$-axis left) and "negative predictive values" (NPV=[true negative by GT]/[total negative by model], solid blue circles, $y$-axis left), of the model's ratings, are graphed versus the pSim threshold value that was applied by the model ( $x$-axis). Also displayed in panel 2 (y-axis right) are the model's "true positive capture rate" (TPCR, dotted red triangles) and "true negative capture rate" (TNCR, dotted blue circles), defined respectively as TPCR=[true positive (TP) by GT]/[total positive by GT (number bold red from panel 1)] and TNCR=[true negative (TN) by GT]/[total negative by GT (number bold blue from panel 1)]. In panels $\mathbf{3}$ (lower left) and $\mathbf{4}$ (lower right), respectively, the number of false positive (FP by GT) and false negative (FN by GT) cases rated by the model at each pSim threshold value (x-axis), are shown stratified by dataset (CheXpert, MIMIC, or $\mathrm{NIH}$; total number cases positive or negative 
207 by the model in parentheses), with the "optimal", lowest pSim threshold achieving $100 \%$ PPV or 208 NPV, as indicated (bold green triangles).

209

210 

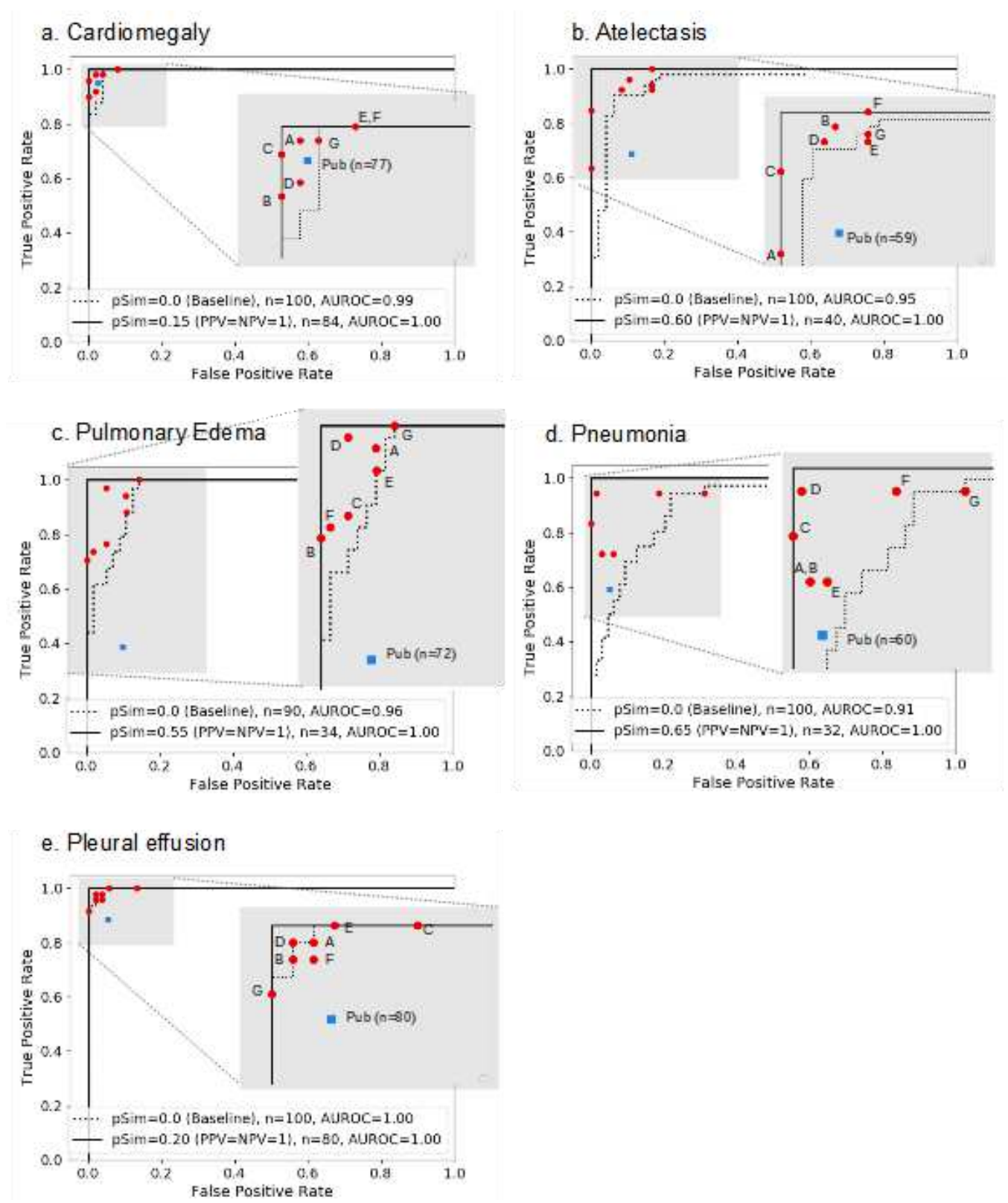

Fig. 3 | AUROC performance of automated-labeling model at two different pSim threshold values, compared to sensitivity, specificity of individual expert radiologists and pooled public labels from three open-source CXR datasets. AUROC performance of our XAI CXR auto-labeling model applied to the CheXpert, MIMIC, and NIH open source datasets, is shown for each of the five labeled imaging features - a cardiomegaly, $\mathbf{b}$ atelectasis, $\mathbf{c}$ pulmonary edema, $\mathbf{d}$ pneumonia, and e pleural effusion. Comparison is to the performance of the individual expert radiologists (A-G, red circles), as well as to the performance of the pooled external annotations (blue squares, $n=n u m b e r$ available labeled external cases per feature). ROC curves ( $y$-axis sensitivity, $x$-axis 1 -specificity) are shown for both the "baseline" $p S i m=0$ threshold (magnified box) and the "optimal" pSim threshold (i.e., the lowest pSim threshold achieving 100\% accuracy, as per Fig. $\mathbf{2}$ panels $\mathbf{3}$ and $\mathbf{4}$ ). 
a. Cardiomegaly: $39 / 50(78 \%)$ positive cases with complete agreement
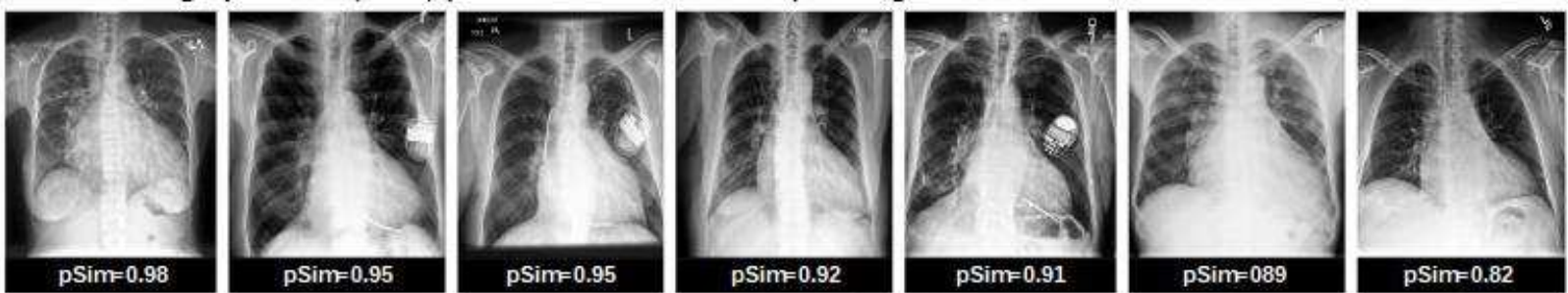

223
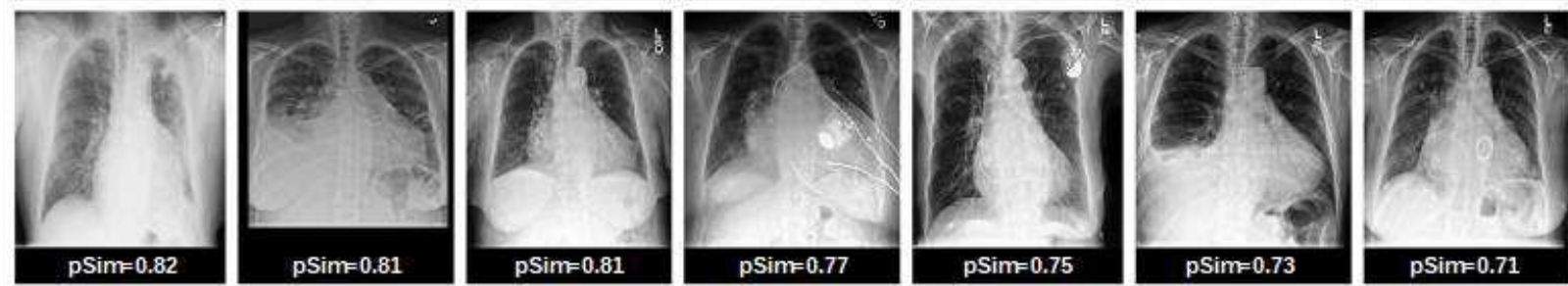

b. Atelectasis: $23 / 50(46 \%)$ positive cases with complete agreement
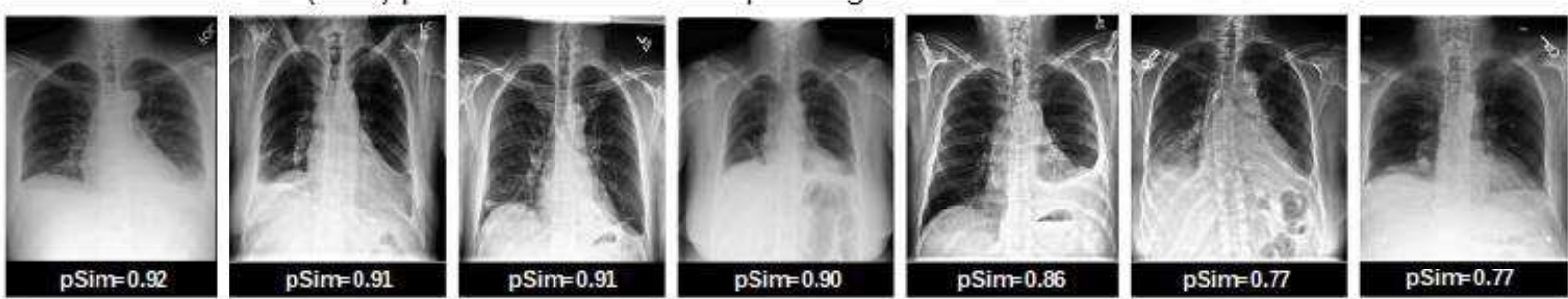

224


c. Pulmonary Edema: $17 / 40$ (43\%) positive cases with complete agreement
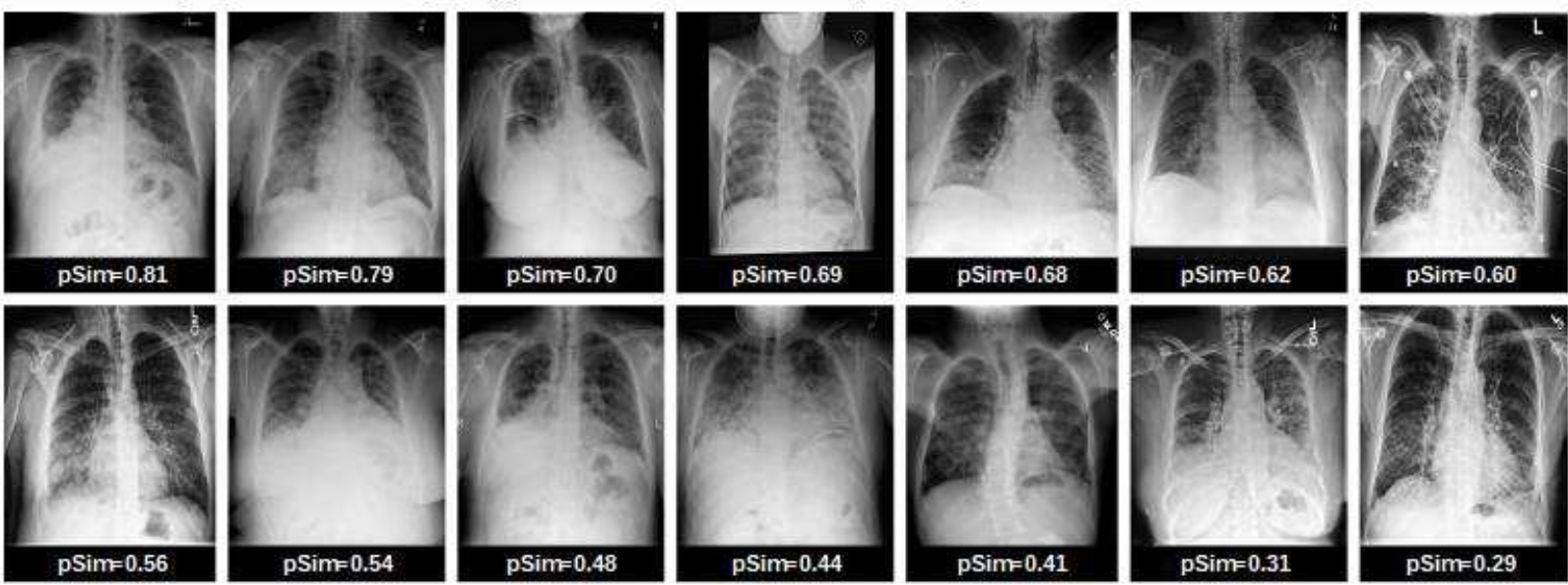

226 



e. Pleural Effusion: $39 / 50(78 \%)$ positive cases with complete agreement
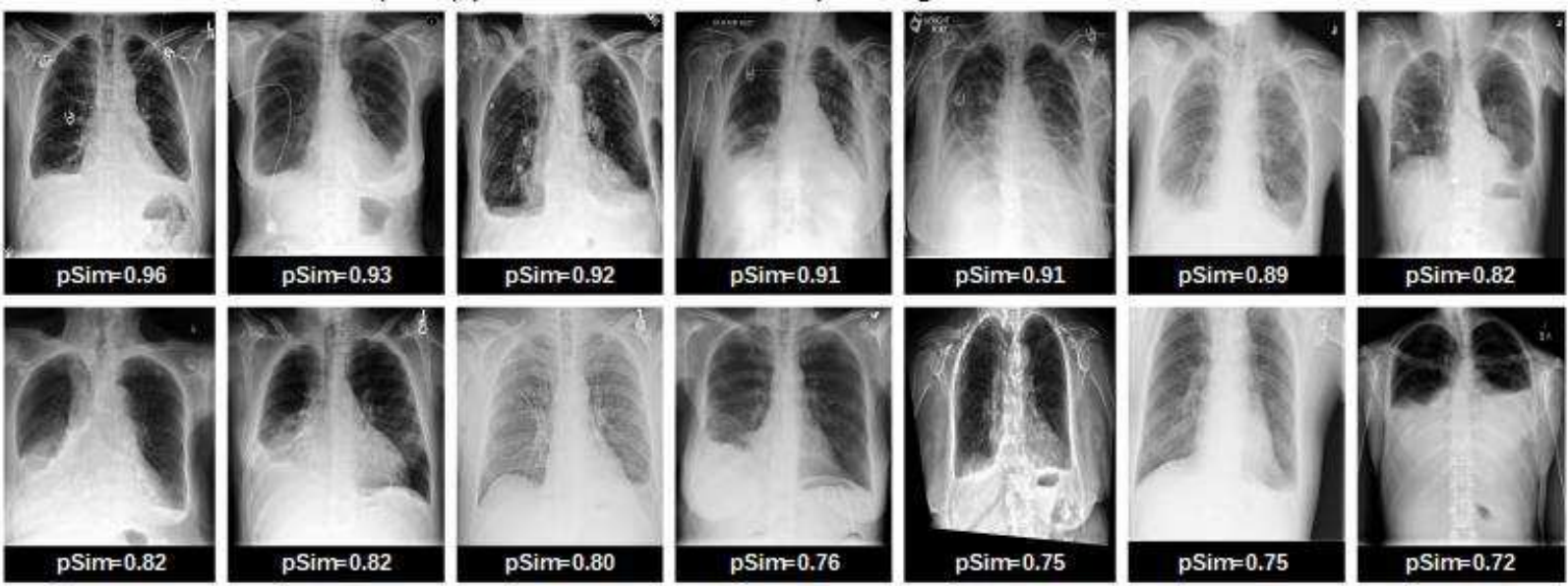

Fig. 4 | Sample PA CXR images of positive examinations identified by the automatedlabeling model, which had complete agreement between all 7 expert radiologists and the XAI model output, for each of the five features studied. Shown are fourteen sample PA CXR images with positive findings, for each of the five features studied (cardiomegaly $\mathbf{a}$, atelectasis $\mathbf{b}$, pulmonary edema $\mathbf{c}$, pneumonia $\mathbf{d}$, and pleural effusion $\mathbf{e}$ ), for which there was complete agreement between both the xAl model output and the 7 expert radiologist interpretations. The pSim threshold values used by the model for auto-labeling feature detection are shown (a-e). For pneumonia, $\mathbf{d}$, there were only 14 positive examinations, out of 50 total positive examinations identified by the model, which had complete agreement with all readers $(14 / 50=28 \%)$, as per Fig. 2.1; percent agreement for the other features is as shown in the above captions (a-c, e). 


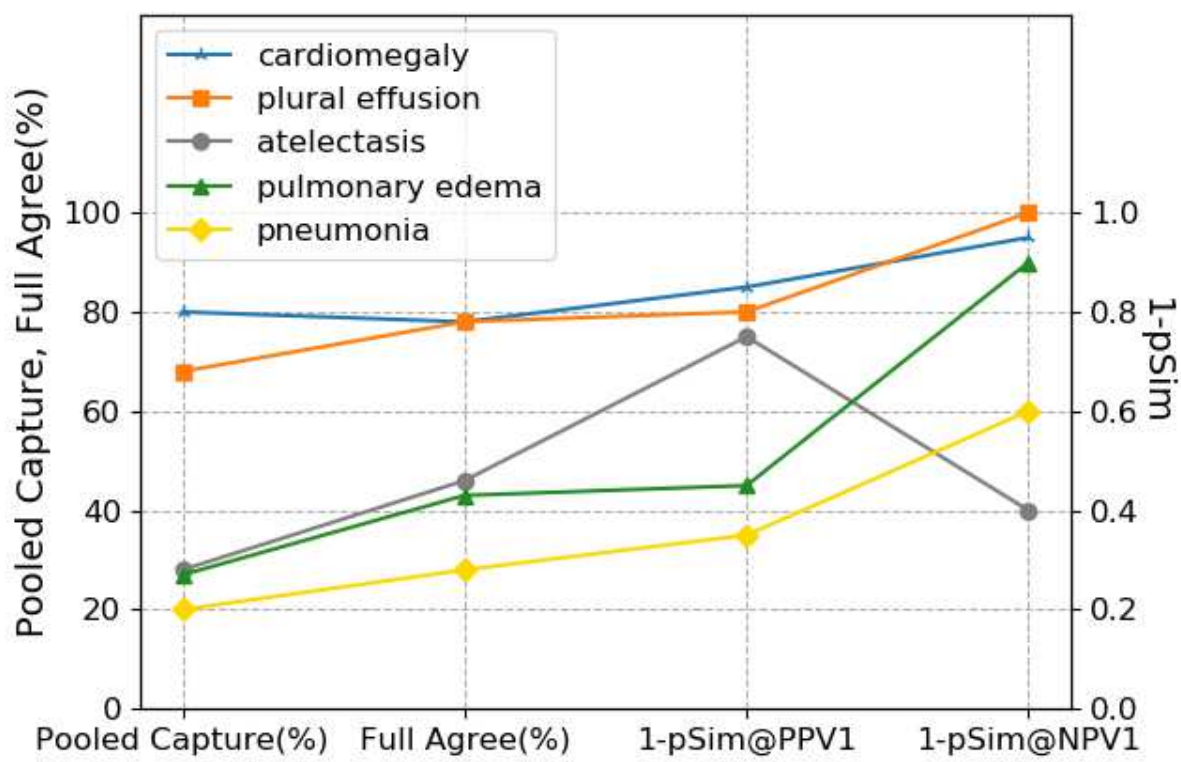

Fig. 5 | Comparison of labeling efficiency/confidence metrics for each of the $\mathbf{5}$ features. For each of the five auto-labeled features - cardiomegaly (blue), pleural effusion (orange), atelectasis (gray), pulmonary edema (green), and pneumonia (yellow) - we compared: (i) the percent of positively auto-labeled CXR's "captured" from the three pooled, full public datasets (i.e. "Pooled 245 Capture\%", from Table 1, far right); (ii) the percent of cases with complete agreement between the model and all 7 expert readers (i.e. "Full Agree\%", from Fig. 4); (iii) the lowest pSim value such that PPV=1 (graphed as "1-pSim", from Fig. 2, panel 3), and (iv) the lowest pSim value such that NPV=1 (graphed as "1-pSim", from Fig. 2, panel 4). Features with higher y-axis values (e.g. cardiomegaly, pleural effusion) correspond to those with greater model auto-labeling efficiency/confidence; features with lower y-axis values (e.g. pneumonia, pulmonary edema) correspond to those with lesser model auto-labeling efficiency/confidence. Of note, in the graph for atelectasis, "1-pSim@PPV1" is higher than "1-pSim@NPV1", which can be interpreted as greater confidence that the model is correct in "ruling-in" the feature (i.e. correctly auto-labeling true-positives) than in "ruling-out" the feature (i.e. correctly auto-labeling true-negatives); this relationship is reversed for the other 4 features (e.g. greater confidence that the model can correctly "rule-out" than "rule-in" pneumonia or pulmonary edema). 


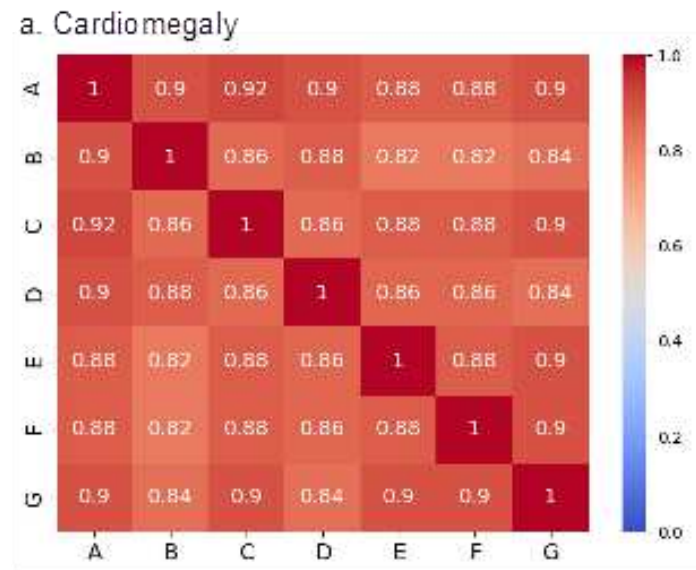

b. Pleural effusion
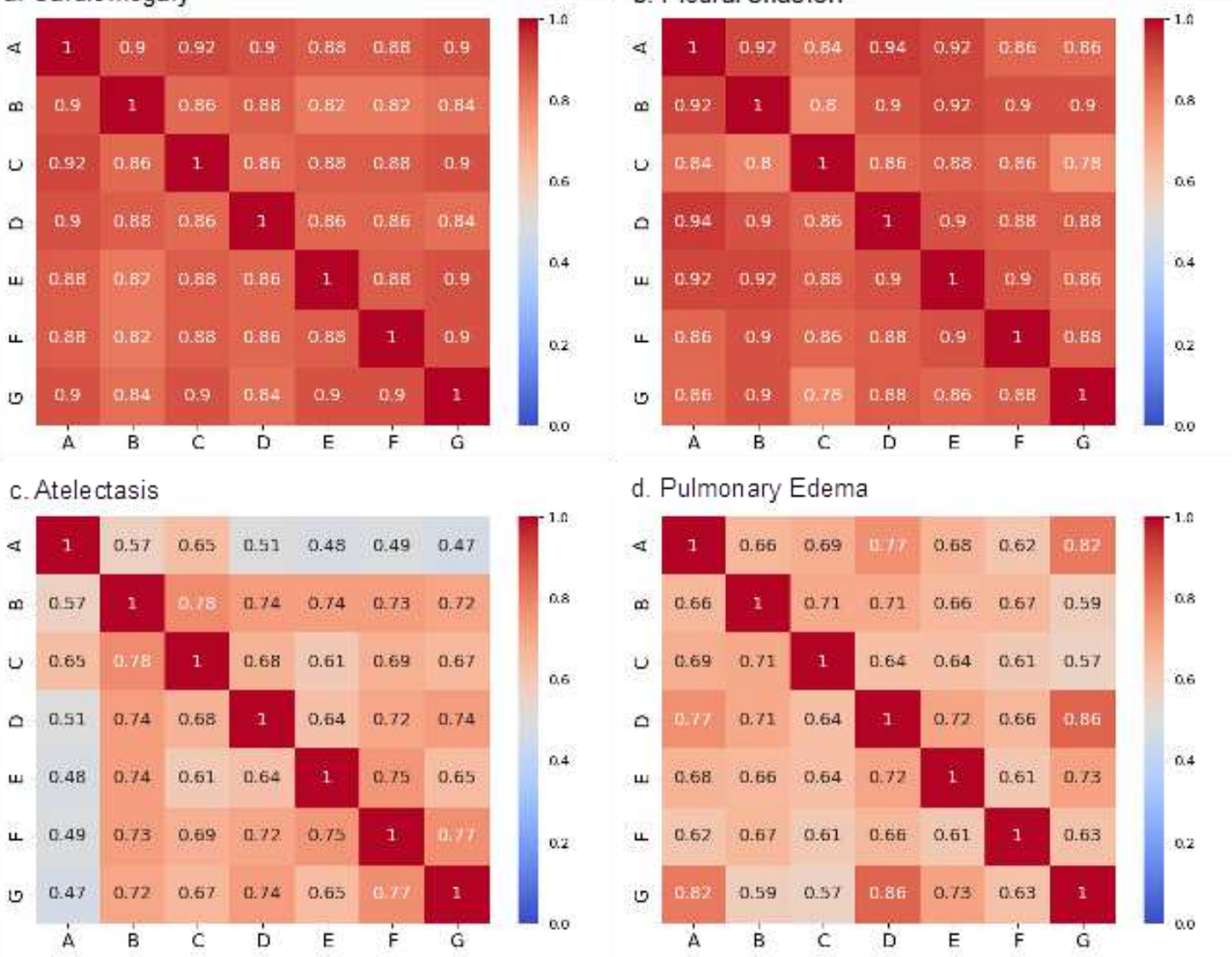

d. Pulmonary Edema
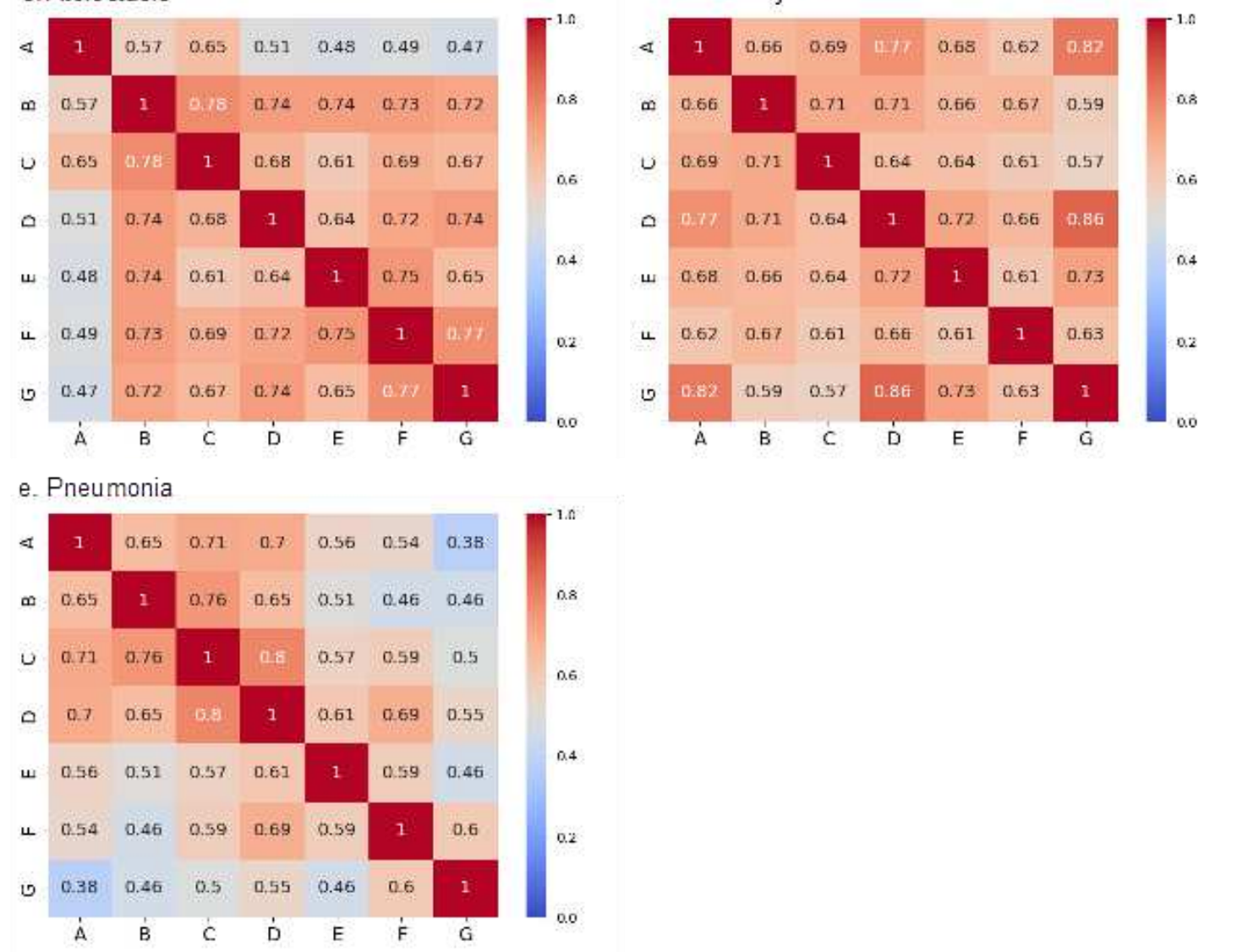

Fig. 6 | Pairwise kappa statistics between the 7 expert radiologists, for each of the 5 features. For each of the five auto-labeled features - cardiomegaly, pleural effusion, atelectasis, pulmonary edema, and pneumonia - the pairwise kappa statistics estimating inter-observer variability are shown in the respective color-coded matrices [11]. 


\begin{tabular}{|l|r|r|r|r|r|r|r|r|c|}
\hline \multicolumn{4}{|c|}{ CheXpert (public labels) } & \multicolumn{4}{|c|}{ Model (auto-labels) for pSim value (PPV,NPV=1) } \\
\hline & \multicolumn{1}{|c|}{ POS } & NEG & unlabeled & $\begin{array}{c}\text { Total } \\
\text { \# PA CXR's }\end{array}$ & pSim & POS & NEG & $\begin{array}{r}\text { \# CXR's } \\
\text { Labeled }\end{array}$ & $\begin{array}{c}\text { Capture } \\
\text { Rate (\%) }\end{array}$ \\
\hline Cardiomegaly & 2,909 & 3,188 & 23,323 & 29,420 & 0.15 & 3,532 & 15,862 & 19,394 & 66 \\
\hline Atelectasis & 3,195 & 271 & 25,954 & 29,420 & 0.60 & 3,150 & 3,654 & 6,804 & 23 \\
\hline Edema & 1,709 & 4,357 & 23,354 & 29,420 & 0.55 & 32 & 5,776 & 5,808 & 20 \\
\hline Pneumonia & 1,198 & 858 & 27,364 & 29,420 & 0.65 & 1,076 & 1,684 & 2,760 & 9 \\
\hline Pleural Effusion & 8,078 & 9,583 & 11,759 & 29,420 & 0.20 & 4,490 & 13,517 & 18,007 & 61 \\
\hline
\end{tabular}

\begin{tabular}{|c|c|c|c|c|c|c|c|c|c|c|c|c|}
\hline \multicolumn{5}{|c|}{ MIMIC (public labels) } & \multicolumn{5}{|c|}{ Model (auto-labels) for pSim value (PPV,NPV=1) } & \multicolumn{3}{|c|}{ Pooled model labels for the 3 public datasets } \\
\hline & POS & NEG & unlabeled & $\begin{array}{c}\text { Total } \\
\text { \# PA CXR's }\end{array}$ & pSim & POS & NEG & $\begin{array}{l}\text { \# CXR's } \\
\text { Labeled }\end{array}$ & $\begin{array}{l}\text { Capture } \\
\text { Rate }(\%)\end{array}$ & $\begin{array}{c}\text { Total } \\
\text { \# PA CXR's }\end{array}$ & $\begin{array}{l}\text { \# CXR's } \\
\text { Labeled }\end{array}$ & $\begin{array}{c}\text { Weighted } \\
\text { Capture Rate (\%) }\end{array}$ \\
\hline Cardiomegaly & 7,427 & 6,124 & 57,672 & 71,223 & 0.15 & 5,536 & 50,347 & 55,883 & 78 & 167,953 & 134,076 & 80 \\
\hline Atelectasis & 7,328 & 376 & 63,519 & 71,223 & 0.60 & 4,052 & 14,960 & 19,012 & 27 & 167,953 & 47,436 & 28 \\
\hline Edema & 2,032 & 9,084 & 60,107 & 71,223 & 0.55 & 30 & 19,585 & 19,615 & 28 & 167,953 & 45,660 & 27 \\
\hline \begin{tabular}{|l|} 
Pneumonia \\
\end{tabular} & 4,793 & 11,660 & 54,770 & 71,223 & 0.65 & 2,081 & 10,980 & 13,061 & 18 & 167,953 & 33,308 & 20 \\
\hline Pleural Effusion & 9,275 & 10,312 & 51,636 & 71,223 & 0.20 & 5,987 & 42,240 & 48,227 & 68 & 167,953 & 114,230 & 68 \\
\hline
\end{tabular}

\begin{tabular}{|l|r|r|r|r|r|r|r|r|r|}
\hline & \multicolumn{4}{|c|}{ NIH (public labels) } & \multicolumn{3}{|c|}{ Model (auto-labels) for pSim value (PPV,NPV=1) } \\
\hline & \multicolumn{1}{|c|}{ POS } & NEG & unlabeled & $\begin{array}{c}\text { Total } \\
\text { \# PA CXR's }\end{array}$ & pSim & POS & NEG & $\begin{array}{c}\text { \# CXR's } \\
\text { Labeled }\end{array}$ & $\begin{array}{c}\text { Capture } \\
\text { Rate (\%) }\end{array}$ \\
\hline Cardiomegaly & 1,563 & 65,747 & 0 & 67,310 & 0.15 & 875 & 57,924 & 58,799 & 87 \\
\hline Atelectasis & 5,728 & 61,582 & 0 & 67,310 & 0.60 & 3,094 & 18,526 & 21,620 & 32 \\
\hline Edema & 276 & 67,034 & 0 & 67,310 & 0.55 & 93 & 20,144 & 20,237 & 30 \\
\hline Pneumonia & 630 & 66,680 & 0 & 67,310 & 0.65 & 1,828 & 15,659 & 17,487 & 26 \\
\hline Pleural Effusion & 6,589 & 60,721 & 0 & 67,310 & 0.20 & 7,615 & 40,381 & 47,996 & 71 \\
\hline
\end{tabular}

Table 1 | Automated-labeling model CXR "capture rate" applied to the three complete public datasets, at the optimized pSim threshold value for maximal accuracy per feature source CXR datasets: CheXpert, MIMIC, and NIH. Dataset composition and number of positively labeled, negatively labeled, and unlabeled PA CXR's for each of the five features (cardiomegaly, atelectasis, edema, pneumonia, and pleural effusion) are shown (Table 1, left). The minimal "optimal" pSim threshold value, such that PPV, NPV $=1$ for labeling accuracy, was selected as per Fig. 2 (Table 1, middle column). It is noteworthy that the model's mean CXR "capture rates" for the pooled results from the three full public datasets (Table 1, far right), closely correspond to those shown in the graphs of Fig. 2.2 (a-e), for the subset of examinations ( $n=90-100)$ labeled by both the model and the expert radiologists. 


\section{Discussion}

278 Accurate, efficient annotation of large medical imaging datasets is an important limitation in the 279 training, and hence widespread implementation, of artificial intelligence (AI) models in healthcare 280 [12-21]. To date, however, there have been few attempts described in the literature to automate 281 the labeling of such large, open-access databases [2-6]. One approach, for example, focused on 282 developing new Al models using labor-intensive, manually annotated subsets of the external 283 datasets, and applying these models to the remaining database [6]. In this study, we demonstrate 284 a method for standardized, automated labeling based on similarity to a previously validated 285 explainable Al model (XAI), using an atlas-based approach, for which the user can specify a 286 quantitative threshold for a desired level of accuracy, the "probability-of-similarity" (pSim) metric. 287 Specifically, we applied our existing Al model for detection of five different CXR features 288 (cardiomegaly, pleural effusion, atelectasis, pulmonary edema, and pneumonia), to three large 289 public open-source datasets (CheXpert, MIMIC, and NIH), and compared the resulting labels to 290 those of 7 human expert radiologists.

292 We showed that our XAI model, by calculating the pSim values for each feature based on 293 comparison to its "remembered" training-set derived reference atlas, could automatically label a 294 subset of the external data at a user-selected, arbitrarily high level of accuracy, equaling or exceeding that of the human experts (Fig. 3).

297 As shown in Fig. 2, the pSim value used for annotation reflects a trade-off between the accuracy 298 of image labeling (i.e., the higher the pSim value, the more accurate the labels) and the efficiency 299 of image labeling (i.e., the higher the pSim value, the fewer examinations that the model selects 300 for annotation). 
302 To evaluate the efficiency of our automated-labeling approach, we applied our xAl model to the 303 three full public datasets, and compared the five auto-labeled features according to the following 304 parameters: (a) the percent of positively auto-labeled CXR's from the three pooled public datasets 305 (i.e., the "capture rate"), (b) the percent of cases with complete agreement between the model 306 and all 7 expert readers, (c) the lowest pSim value for annotation such that all positive cases 307 captured are "true positive" (i.e., "optimal" pSim for PPV=1), and (d) the lowest pSim value for 308 annotation such that all negative cases captured are "true negative" (i.e., "optimal" pSim for $309 \mathrm{NPV}=1)$. We found a strong correlation between the magnitude of these parameters for each of 310 the annotated features, as shown in Fig. 5. It is noteworthy that the positive "capture rates" from 311 the three pooled public datasets also strongly correlated with the "capture rates" graphed in Fig. 312 2.2, for the subset of examinations $(n=90-100)$ labeled by both the model and the radiologist 313 experts. Moreover, the parameter values reported for each feature corresponded well with the 314 kappa values for inter-observer variability shown in Fig.6.

316 Together, these results suggest that the overall accuracy and efficiency of the auto-labeling model, 317 applied to the full public datasets at the "optimal" pSim for each feature, is similar to the accuracy 318 and efficiency of the model as applied to the subset of examinations annotated by the 7 expert 319 radiologists. These results also suggest greater auto-labeling efficiency, with higher confidence 320 in label accuracy, for "cardiomegaly" and "pleural effusion" - two of the more objective findings in 321 CXR interpretation - and lesser auto-labeling efficiency, with lower confidence in label accuracy, 322 for "pneumonia" and "pulmonary edema" - two of the more subjective assessments in CXR 323 interpretation. Indeed, the larger the quantity "1-pSimoptimal" for a given feature (where $0 \leq \mathrm{pSim} \leq 1$ 324 and pSim ${ }_{\text {optimal }}=$ the minimum $\mathrm{pSim}$ value such that PPV/NPV=1), the more reliable and robust is 325 the labeling for that feature, based on similarity to the "remembered" reference atlas derived from 326 the model's NLP training set. 
A noteworthy aspect of our approach relates to system deployment. We can apply the pSim value

329 threshold to each class independently, selecting a "low" pSim value for high conspicuity features 330 with high inter-rater agreement, and selecting a "high" pSim value for noisier, more subjective 331 non-specific features with lower inter-rater agreement, the latter at the cost of generating fewer 332 labeled examinations (i.e., lower "capture rate"). Employing pSim values helps quantify which 333 features of the Al model are most reliably annotated and which need to be improved, making it 334 possible to measure system robustness. Deploying the XAI system is also HIPAA compliant, as 335 no patient identifiable source data need be stored, since the mode selection (Fig. 1) uses only the 336 encoded predicted probability distributions for categories and the compressed information from 337 the UMAP transformation [22] for the atlas.

Another technical capability of our system is the "re-annotation" mode, which was not used in the current study, but which may be of value in real-world clinical settings. This mode is a component

341 of the "explainability" functionality of our model, by which the system can prompt or query a human 342 user if the pSim value for feature detection falls below a pre-selected threshold. More generally, 343 the re-annotation mode has the potential to be applied to other medical Al models as a safety 344 feature, alerting users that there is a measurable, quantitative probability of interpretive error in 345 the Al model's output.

347 Other current approaches to auto-labeling have involved semi-supervised [6, 23] and self348 supervised [24-27] learning. Because these approaches assume low correlation between classes, 349 however, their performance has not been validated for multi-label CXR classification models with 350 high interclass correlation. Transfer learning and fine-tuning have also been attempted to improve 351 performance when independently developed models are applied to external datasets [28-30], 352 however, these methods are often impractical because different institutions are likely to use 353 different definitions for similar categories, and capturing data with external labels based on even 
354 slightly different definitions can introduce considerable noise when such data is used for training

355 or re-training new models. Our approach, however, allows for generation of standardized labels,

356 with a user defined probability of similarity to that of established models. Our atlas-based

357 approach, which simplifies the computational issues by focusing on small patch regions with lower

358 interclass and higher intraclass correlations, was capable of achieving high accuracy and

359 efficiency for auto-labeling three large public open source CXR datasets, similar to or exceeding

360 that of human experts.

362 Our auto-labeling Al model reflects several characteristics of human intelligence [31] in general, 363 and radiologist-mimicking behavior in particular. Specifically, our system is "smart", in that it can 364 access its "memory" of examination features present in the training set, and quantitatively 365 estimate their similarity to features in the new, external examination data. The "1-pSimoptimal" 366 metric for each feature provides a measure of the "intelligence" of the system for efficient accurate 367 labeling, and its value (between 0 and 1) reflects the quality (i.e., ground-truth accuracy) of the 368 NLP-derived dataset used for initial training. The model can also provide feedback to users 369 through its "explainability" functionality, by displaying examples of the features under 370 consideration from its reference atlas together with their associated pSim value; this interaction 371 offers the user an additional level of confidence that the model is doing what it's supposed to do.

372 In this regard, our system can be viewed as an "augmented" intelligence tool to improve the 373 accuracy and efficiency of medical imagers.

375 Indeed, one limitation of our model is that its labeling accuracy and efficiency is directly 376 proportional to the quality of the initial training set. This may help explain why cardiomegaly and 377 pleural effusion - two high-conspicuity features routinely correctly described in the radiology 378 reports identified by NLP for model training - have higher efficiency metrics (Fig. 5) than 379 pulmonary edema and pneumonia, which are more non-specific and variably assessed by 
different radiologists. This also may help explain why the "1-pSimoptimal values for NPV=1" in Fig.5



382 atelectasis is a lower conspicuity, more non-specific feature typically noted in CXR radiology

383 reports only when it is present, but not mentioned when it is absent (i.e., the model "learned" from

384 its NLP derived training set to have a higher level of certainty, and hence a higher "1-

385 pSimoptimal" value, when atelectasis is present, then when it is absent). Pulmonary edema and

386 pneumonia, on the other hand, are typically described in CXR reports with a higher level of

387 certainty when they are definitely absent (e.g., "no evidence of pulmonary edema or pneumonia"),

388 than when they are possibly present (e.g., "cannot exclude pulmonary edema or pneumonia").

Another limitation of our model is that our proposed xAI system requires substantial computational resources and storage space to provide the prediction basis and to operate the mode selection module. Because the explainable modules have been designed to operate independently, however, we can differentially deploy the xAl system of adjusted capabilities according to the specification of a given server.

In summary, we have developed and demonstrated an explainable Al model for automated397 labeling of five different CXR imaging features, at a user selected quantitative level of confidence, 398 based on similarity to the reference atlas library of an existing, validated model. The ability to 399 automatically, accurately, and efficiently annotate large medical imaging datasets may be of 400 considerable value in developing important, high-impact AI models that bring added value to, and 401 are widely accepted by, the healthcare community. This approach might not only benefit re402 training to improve the accuracy of existing Al models, but also help to standardize labeling of 403 open-source datasets, for which the provided labels can be noisy, inaccurate, or absent. Such 404 standardization may, in turn, reduce the amount of data required for accurate model building, 405 facilitating training and re-training from initial small, but well annotated, datasets. 
408 This study was compliant with the Health Insurance Portability and Accountability Act and was

409 approved by the Institutional Review Board of the Massachusetts General Hospital.

411 Retrospective collection of the development and test datasets. The development dataset 412 contained CXR images acquired between February 2015 and February 2019. All DICOM (digital 413 imaging and communications in medicine) images were de-identified before data analyses. To 414 make a consistent dataset, we chose only examinations that had associated radiology reports, 415 view position information (e.g. AP/PA projections, "portable", etc.), and essential patient identifiers 416 (including but not limited to medical record number, age, or gender). If an examination had 417 multiple CXR images, only a single CXR image was included. We randomly selected 1000 images 418 for each view position as a test set; the remaining examinations, from non-overlapping patients, 419 were separated into training and validation sets (Supplementary Fig. 1).

421 Labeling of the development and test datasets. The labels for the training and validation sets 422 were determined exclusively from the automated NLP assignments, whereas those for the test 423 set were determined by consensus of three U.S. board-certified radiologists at our institution 424 (further details provided in Supplementary Table 1) using the "Mark-it" tool 425 (https://markit.mgh.harvard.edu, MA, USA) for annotation [9].

427 Network training. Densely Connected Convolutional Network (DenseNet-121) [32], which 428 connects each layer to all other layers in a feed-forward method, was selected to develop the 20 429 pathologic features detection and classification system. The pretrained model, available from the 430 official repository in Pytorch [33, 34], was fine-tuned with our training dataset after the last fully 431 connected layer with 1000 outputs and the first convolutional layer were replaced with 21 outputs 
432 (i.e., 20 pathologic features and view position) and with inputs of 1 channel depth, respectively. 433 The network topology was optimized using AdamW [35], where we used a batch size of 144, a 434 learning rate of $1 \times 10^{-4}$, beta- 1 of 0.9 , beta- 2 of 0.999 , epsilon of $1 \times 10^{-8}$, and weight decay of $4351 \times 10^{-5}$. In the training step, real-time data augmentation was performed by applying geometric 436 transformations: rotation from -10 to 10 , scaling to $110 \%$, random crop to $512 \times 512$, random 437 horizontal flip with $1 \%$ probability. All experiments were conducted on four GPUs of Tesla V100 438 SXM 32 GB [NVIDIA DGX, CA, USA], and all deep-learning models were implemented with 439 Pytorch (v.1.2.0).

441 Weighted loss function. The Binary Cross-Entropy (BCE) loss function was weighted by the 442 ratios of positive and negative samples for each class label $\left(\alpha_{P}^{c}\right.$ and $\left.\alpha_{N}^{c}\right)$, in a similar fashion as 443 described previously [4], for multi-label classification. We considered two additional weights: the 444 first weight had to reflect the ratio of the number of effective samples ( $\alpha_{s}^{c}$, the maximal sum 445 number between positive and negative labels among 20 features divided by that of the $\mathrm{c}$-th feature)

446 to train because of consideration of ignore labels for each feature. When training the Al model, 447 we experimentally found that using samples with the other view position as well as those with a 448 targeted view position can improve the generalization performance of the model, so we added 449 the second weight $(\alpha(v))$ in the loss function to relatively control the impact of samples with the 450 target view position. The weighted BCE loss function is given by the equation (1):

$$
L_{W-B C E}(x, y, t, v)=-\alpha(v) \sum_{c=1}^{J} \alpha_{s}^{c}\left\{\alpha_{P}^{c} t^{c} \ln y^{c}+\alpha_{N}^{c}\left(1-t^{c}\right) \ln \left(1-y^{c}\right)\right\},
$$

452 where $x$ denotes CXR images, the model's output is $y=\left\{y^{1}, y^{2}, \ldots, y^{J}\right\}$ that indicates the 453 predicted probability of $J$ classes, $v$ is a view position of the image, and $t=\left\{t^{1}, t^{2}, \ldots, t^{J}\right\}$ means 454 the labels of features extracted by NLP. In addition, $\alpha_{s}^{c}$ is defined as $\left(\left|P^{m}\right|+\left|N^{m}\right|\right) /\left(\left|P^{c}\right|+\left|N^{c}\right|\right)$ 455 in order to make fairness among classes with different numbers of effective samples which 456 consider only ' 0 ' and ' 1 ', not ' -1 '. Here, $\left|P^{c}\right|$ and $\left|N^{c}\right|$ are the total numbers of ' 1 's and ' 0 's in labels 
457 for $c$ feature, and $m$ means the class index having the maximum total number of both ' 1 's and '0's

$458 \quad\left(m=\arg \max _{c}\left(\left|P^{c}\right|+\left|N^{c}\right|\right)\right)$. We also define $\alpha_{P}^{c}=\frac{\left|P^{c}\right|+\left|N^{c}\right|}{\left|P^{c}\right|}$ and $\alpha_{N}^{c}=\frac{\left|P^{c}\right|+\left|N^{c}\right|}{\left|N^{c}\right|}$ for solving the 459 imbalance between positive and negative; $\alpha(v)$ is set to $\omega$ if $v$ is the targeted view, 1 for the others.

Design overview for quantitative, explainable, atlas-based system. Our automated dataset labeling, based on similarity to a validated CXR AI model, requires calculation of two quantitative atlas-based parameters, the "patch similarity" and "confidence" probabilities (values between 0 and 1), as per Fig. 1. For the "patch similarity" computation, a patch atlas is generated based on class activation mapping (CAM) [7, 8]; for the "confidence" computation a distribution atlas is generated based on predicted probabilities (Fig. 1a,b). The harmonic mean between the patch similarity and confidence values are then used to calculate a "probability of similarity" ( $p$ Sim) for 468 each feature (Fig. 1c).

Predicted probabilities, model ensemble, and distribution atlas creation. To improve the

471 robustness of the entire system, an ensemble of six DenseNet-121 models is composed using 472 unweighted averaging, such that the final probability is determined as an average of probabilities 473 predicted by the six models [36]. Those six models are constructed by independently training with 474 three weights (i.e., $\omega=1.1,1.5$, and 2.0 in $\alpha(v)$ ) for PA view, then selecting two models maximized by AUROC and accuracy, respectively. To create the Distribution-atlas, we do inference with the

476 trained Al model on a full training dataset, to obtain two probability distributions of positive and 477 negative samples for the training dataset. These probability distributions are saved as the 478 Distribution-atlas for each feature.

480 Patch atlas creation based on CAM ensemble method. To improve the localization 481 performance of our class activation mapping, we developed an ensemble method as follows: by 
removing noise components of a single CAM, adding only significant components, and 483 normalizing it in equation (2), the ensemble CAM was able to highlight sharply the overlapping 484 regions among the single CAMs.

$$
\operatorname{CAM}_{E}^{c}=\operatorname{Normalize}\left(\sum_{S=1}^{S} C A M_{S}^{c} \odot U_{\tau}\right),
$$

where $C A M_{E}^{c}$ means the ensemble CAM matrix, $C A M_{S}^{c}$ is a CAM matrix for the c-class generated

487 from s-th single model, and $S$ denotes the number of models. $U_{\tau}$ denotes a matrix with the component of $u_{i, j}=u\left(C A M_{S}^{c}(i, j)-\tau\right)$ to determine CAM values less than $\tau$ as noise components 489 and to remove them. $u$ is a unit step function, $\odot$ means the Hadamard product, and Normalize is 490 a linear scale for converting into a standard range between 0 and 1.

492 To create the patch atlas, we search for main contours on a high-resolution CAM (512x512) 493 generated from a CAM for each class, select a bounding box to include the outline, define it as 494 the patch, and save it (one or two patches from a CAM are considered in this study). For each feature, patches are saved as typical, representative patterns from only the CXR images with the

496 Al model's predicted probability of being greater than or equal to 0.9 . We train a cosine metric 497 based UMAP model using the patches for all features [22]. The UMAP model transforms the 498 patches into coordinates in two-dimensional embedding space, such that the smaller the 499 Euclidean distance in this space, the higher the cosine similarity. For the automated labeling 500 method, therefore, the patch atlas consists of coordinates for all patches in the two-dimensional 501 embedding space and the UMAP model (Fig. 1b).

503 Patch similarity value calculation. To calculate the patch similarity as shown in Fig. 1b, we 504 need to extract the Prediction-basis $\left(\Psi_{p b}^{c}\right)$ for the c-th feature by calculating Euclidean distance 505 between the UMAP transformed coordinate of the test image and the Patch-atlas, and then by 506 selecting K-basis with the minimum distance as equation (3): 


$$
\Psi_{p b}^{c}=\left\{\Omega_{p b}^{c}(1), \ldots, \Omega_{p b}^{c}(K)\right\}
$$

508 where $\Omega_{p b}^{c}(k)$ denotes the patch with the $k$-th minimum Euclidean distance among the Patch-

509 atlas, and the Euclidean distance is calculated by $\| f_{U M A P}^{c}\left(y_{p}^{c}\right)-\left.A_{P-U M A P}^{c}(i)\right|_{2}$ for $i=$ $5101, \ldots, n\left(A_{P-U M A P}^{c}\right)$. Moreover, $f_{U M A P}^{c}$ is the trained UMAP model for c-class, $y_{p}^{c}$ is a 1024-

511 dimensional patch vector calculated by a test image, $A_{P-U M A P}^{c}$ is the Patch-atlas, and $n\left(A_{P-U M A P}^{c}\right)$

512 is the size of the Patch-atlas. The patch similarity is proposed to enable the Al model to interpret

513 the new patch based on the prediction-basis $\left(\Psi_{p b}^{c}\right)$, as a quantitative metric. The metric is

514 calculated by a percentile of how close a patch of a test image is on a prediction-basis of $\mathrm{K}$ 515 patches in the embedding space.

$$
\text { patch similarity }=1-f_{D}^{c}\left(\frac{1}{K} \cdot \sum_{m=1}^{K}|| f_{U M A P}^{c}\left(y_{p}^{c}\right)-\left.f_{U M A P}^{c}\left(\Omega_{p t b}^{c}(m)\right)\right|_{2}\right) \text {, }
$$

517 where $f_{D}^{c}$ denotes a function calculating a percentile for the mean Euclidean distance of K-nearest 518 patches for the test image, based on a distribution of the mean Euclidean distance for all patches 519 of the Patch-atlas.

521 Confidence value calculation. As per Fig. 1b, we propose the confidence metric, based on the 522 distribution atlas, as a measure of the trust level between the positive and negative predicted 523 probabilities for a feature. This quantitative metric is simply defined with equations (5) and (6) for 524 positive and negative predicted features, as follows:

$$
\begin{aligned}
& \text { Confidence }_{P}=\max \left(f_{P}^{c}\left(y^{c}\right)-\left(1-f_{N}^{c}\left(y^{c}\right)\right), 0\right) \\
& \text { Confidence }_{N}=\max \left(\left(1-f_{N}^{c}\left(y^{c}\right)\right)-f_{P}^{c}\left(y^{c}\right), 0\right)
\end{aligned}
$$

527 Assuming that a predicted probability is $y^{c}$ for c-class, we calculate a percentile $\left(f_{P}^{c}\left(y^{c}\right)\right)$ in the 528 positive Distribution-atlas and a percentile $\left(1-f_{N}^{c}\left(y^{c}\right)\right)$ in the negative Distribution-atlas. Then, 529 the difference between two percentiles is calculated as the confidence. Because the predictive 530 ability of the xAI model for each feature is related to the shape and degree of intersection of the 
531 two probability density curves (positive and negative) on the distribution-atlas, the confidence

532 metric, as defined based on equations (5) and (6), provides a quantitative measure analogous to

533 a p-value between different statistical distributions. In other words, the higher the confidence value

534 for a label, the higher the likelihood that the test image is mapping to the correct label, and the

535 lower the likelihood of incorrect mapping. Moreover, this metric has the ability to quantify different

536 levels of confidence according to different distributions of feature characteristics on the distribution

537 atlas for each class of the model, even at the same predicted probabilities.

539 pSim calculation, pSim threshold selection. Our automated dataset labeling method 540 calculates the pSim value using a harmonic mean between confidence and patch similarity 541 (pSimilarity in equation 7) for each test image.

$$
\mathrm{pSim}=2 \cdot \text { confidence } \cdot \text { pSimilarity } /(\text { confidence }+ \text { pSimilarity })
$$

543 The pSim threshold for each feature is chosen by the lowest pSim values that can achieve $100 \%$ 544 PPV and NPV, as per Fig. 2.

546 An additional functionality of our model design includes a "mode selection" algorithm, which, using 547 the selected pSim threshold value, can determine both the image label (positive, negative, or 548 unlabeled) and a "mode" - self-annotation or re-annotation - or each test image, as per Fig. 1 and 549 Supplementary Table 3. The "re-annotation" mode, which was not applied in this current study 550 but can be used as part of the "explainability" functionality of the model, to prompt the system to 551 alert or query a human user if the pSim value for a feature falls below a selected threshold.

553 Statistical analyses. To assess the statistical significance of the AUROC's, we calculated 95\% 554 Cls using a non-parametric bootstrap approach via the following process: first, 1000 cases were 555 randomly sampled from the test dataset of 1000 cases with replacement, and the DCNN models 556 were evaluated on the sampled test set. After running this process 2,000 times, 95\% Cls were 
557 obtained by using the interval between 2.5 and 97.5 percentiles from the distribution of

558 AUROCs. The $95 \%$ Cls of percentage accuracy, sensitivity and specificity of the models at the 559 selected operating point were calculated using binomial proportion Cls. 


\section{References}

562 [1] Lee, H., Yune, S., Mansouri, M., Kim, M., Tajmir, S.H., Guerrier, C.E., Ebert, S.A., Pomerantz,

563 S.R., Romero, J.M., Kamalian, S., Gonzalez, R.G., Lev, M.H., Do, S. An explainable deep-

564 learning algorithm for the detection of acute intracranial haemorrhage from small datasets. Nature

565 biomedical engineering 3, 173-182, (2019).

566 [2] Irvin, J., Rajpurkar, P., Ko, M., Yu, Y., Ciurea-Ilcus, S., Chute, C., Marklund, H., Haghgoo, B.,

567 Ball, R., Shpanskaya, K. and Seekins, J. Chexpert: A large chest radiograph dataset with

568 uncertainty labels and expert comparison. In Proceedings of the AAAl Conference on Artificial

569 Intelligence 33, 590-597 (2019).

570 [3] Johnson, A.E., Pollard, T.J., Greenbaum, N.R., Lungren, M.P., Deng, C.Y., Peng, Y., Lu, Z.,

571 Mark, R.G., Berkowitz, S.J. and Horng, S. MIMIC-CXR-JPG, a large publicly available database

572 of labeled chest radiographs. Preprint at http://arXiv:1901.07042 (2019).

573 [4] Wang, X., Peng, Y., Lu, L., Lu, Z., Bagheri, M., \& Summers, R. M. Chestx-ray8: Hospital-scale

574 chest $\mathrm{x}$-ray database and benchmarks on weakly-supervised classification and localization of

575 common thorax diseases. In Proceedings of the IEEE conference on computer vision and pattern

576 recognition, 2097-2106 (2017).

577 [5] Bustos, A., Pertusa, A., Salinas, J.M. and de la Iglesia-Vayá, M. Padchest: A large chest x-ray

578 image dataset with multi-label annotated reports. Medical image analysis 66, 101797, (2020).

579 [6] Kim, T.K., Paul, H.Y., Hager, G.D. and Lin, C.T. Refining dataset curation methods for deep

580 learning-based automated tuberculosis screening. Journal of Thoracic Disease, 12(9), 5078-5085,

581 (2020).

582 [7] Zhou, B., Khosla, A., Lapedriza, A., Oliva, A. and Torralba, A. Learning deep features for

583 discriminative localization. In Proceedings of the IEEE conference on computer vision and pattern

584 recognition, 2921-2929, (2016). 
585 [8] Selvaraju, R.R., Cogswell, M., Das, A., Vedantam, R., Parikh, D. and Batra, D. Grad-CAM: 586 Visual explanations from deep networks via gradient-based localization. In Proceedings of the 587 IEEE international conference on computer vision, 618-626, (2017).

588 [9] Witowski, J., Choi, J., Jeon, S., Kim, D., Chung, J., Conklin, J., Longo, M.G.F., Succi, M.D. 589 and Do, S. Marklt: A Collaborative Artificial Intelligence Annotation Platform Leveraging 590 Blockchain For Medical Imaging Research. Blockchain in Healthcare Today, (2021).

591 [10] Powers, D. Evaluation: From Precision, Recall and F-Factor to ROC, Informedness, 592 Markedness \& Correlation. Journal of Machine Learning Technologies, 2(1), 37-63 (2008).

593 [11] Stehman, S.V. Selecting and interpreting measures of thematic classification accuracy.

594 Remote sensing of Environment, 62(1), 77-89, (1997).

595 [12] Lakhani, P., and Sundaram, B. Deep learning at chest radiography: automated classification

596 of pulmonary tuberculosis by using convolutional neural networks. Radiology, 284(2), 574-582, 597 (2017).

598 [13] Baltruschat, I. M., Nickisch, H., Grass, M., Knopp, T., and Saalbach, A. Comparison of deep 599 learning approaches for multi-label chest X-ray classification. Scientific reports 9(1), 1-10, (2019) 600 [14] Pasa, F., Golkov, V., Pfeiffer, F., Cremers, D., \& Pfeiffer, D. Efficient deep network 601 architectures for fast chest X-ray tuberculosis screening and visualization. Scientific reports $\mathbf{9 ( 1 )}$, 602 1-9, (2019).

603 [15] Wang, L., Lin, Z. Q., and Wong, A. Covid-net: A tailored deep convolutional neural network 604 design for detection of covid-19 cases from chest x-ray images. Scientific Reports 10(1), 1-12, 605 (2020).

606 [16] Rajpurkar, P., et al. CheXaid: deep learning assistance for physician diagnosis of tuberculosis 607 using chest x-rays in patients with HIV. NPJ digital medicine 3(1), 1-8, (2020).

608 [17] Oh, Y., Park, S., and Ye, J. C. Deep learning covid-19 features on cxr using limited training 609 data sets. IEEE Transactions on Medical Imaging 39(8), 2688-2700, (2020). 
610 [18] Nam, J.G., Park, S., Hwang, E.J., Lee, J.H., Jin, K.N., Lim, K.Y., Vu, T.H., Sohn, J.H., Hwang,

611 S., Goo, J.M. and Park, C.M. Development and validation of deep learning-based automatic

612 detection algorithm for malignant pulmonary nodules on chest radiographs. Radiology 290(1), 613 218-228, (2019).

614 [19] Sim, Y., Chung, M.J., Kotter, E., Yune, S., Kim, M., Do, S., Han, K., Kim, H., Yang, S., Lee,

615 D.J. and Choi, B.W. Deep convolutional neural network-based software improves radiologist

616 detection of malignant lung nodules on chest radiographs. Radiology 294(1), 199-209, (2020).

617 [20] Sung, J., Park, S., Lee, S.M., Bae, W., Park, B., Jung, E., Seo, J.B. and Jung, K.H. Added

618 Value of Deep Learning-based Detection System for Multiple Major Findings on Chest

619 Radiographs: A Randomized Crossover Study. Radiology, 202818, (2021).

620 [21] Zech, J. R., Badgeley, M. A., Liu, M., Costa, A. B., Titano, J. J., \& Oermann, E. K. Variable 621 generalization performance of a deep learning model to detect pneumonia in chest radiographs:

622 a cross-sectional study. PLoS medicine 15(11), e1002683, (2018).

623 [22] McInnes, L., Healy, J. and Melville, J. Umap: Uniform manifold approximation and projection 624 for dimension reduction. Preprint at http://arXiv:1802.03426 (2018).

625 [23] Berthelot, D., Carlini, N., Goodfellow, I., Papernot, N., Oliver, A., and Raffel, C. A. Mixmatch: 626 A holistic approach to semi-supervised learning. In Advances in Neural Information Processing 627 Systems, 5050-5060, (2019).

628 [24] He, K., Fan, H., Wu, Y., Xie, S. and Girshick, R. Momentum contrast for unsupervised visual 629 representation learning. In Proceedings of the IEEE/CVF Conference on Computer Vision and 630 Pattern Recognition, 9729-9738, (2020).

631 [25] Chen, T., Kornblith, S., Norouzi, M. and Hinton, G. A simple framework for contrastive 632 learning of visual representations. In Proceedings of International conference on machine learning, 633 1597-1607, (2020). 
634 [26] Caron, M., Misra, I., Mairal, J., Goyal, P., Bojanowski, P. and Joulin, A. Unsupervised learning 635 of visual features by contrasting cluster assignments. In Proceedings of Advances in Neural 636 Information Processing Systems (NeurIPS), (2020).

637 [27] Hadsell, R., Chopra, S. and LeCun, Y. Dimensionality reduction by learning an invariant 638 mapping. In Proceedings of the IEEE conference on computer vision and pattern recognition, 639 1735-1742, (2006).

640 [28] Apostolopoulos, I. D., \& Mpesiana, T. A. Covid-19: automatic detection from x-ray images 641 utilizing transfer learning with convolutional neural networks. Physical and Engineering Sciences 642 in Medicine 43(2), 635-640, (2020).

643 [29] Shin, H.C., Roth, H.R., Gao, M., Lu, L., Xu, Z., Nogues, I., Yao, J., Mollura, D. and Summers, 644 R.M. Deep convolutional neural networks for computer-aided detection: CNN architectures, 645 dataset characteristics and transfer learning. IEEE transactions on medical imaging 35(5), 1285646 1298, (2016).

647 [30] Yosinski, J., Clune, J., Bengio, Y., and Lipson, H. How transferable are features in deep 648 neural networks?. Preprint at http://arXiv:1411.1792. (2014)

649 [31] Kolb, D. A. Experiential learning: Experience as the source of learning and development (FT 650 press, 2014)

651 [32] Huang, G., Liu, Z., Van Der Maaten, L. and Weinberger, K.Q. Densely connected 652 convolutional networks. In Proceedings of the IEEE conference on computer vision and pattern 653 recognition, 4700-4708, (2017).

654 [33] Deng, J., Dong, W., Socher, R., Li, L.J., Li, K. and Fei-Fei, L. Imagenet: A large-scale 655 hierarchical image database. In 2009 IEEE conference on computer vision and pattern recognition, 656 248-255, (2009)

657 [34] Paszke, A., Gross, S., Massa, F., Lerer, A., Bradbury, J., Chanan, G., Killeen, T., Lin, Z., 658 Gimelshein, N., Antiga, L. and Desmaison, A. Pytorch: An imperative style, high-performance 659 deep learning library. Preprint at http://arXiv:1912.01703 (2019). 
660 [35] Loshchilov, I. and Hutter, F. Decoupled weight decay regularization. Preprint at 661 http://arXiv:1711.05101 (2017).

662 [36] Ju, C., Bibaut, A. and van der Laan, M. The relative performance of ensemble methods with 663 deep convolutional neural networks for image classification. Journal of Applied Statistics 45, 664 2800-2818, (2018).

665 [37] Langlotz, C. P. RadLex: a new method for indexing online educational materials. http://radlex.org/ 666 (2006).

667 [38] Bird, S., Klein, E. and Loper, E. Natural language processing with Python: analyzing text with 668 the natural language toolkit. (O'Reilly Media, Inc., 2009).

669 [39] Honnibal, M., \& Montani, I. spacy 2: Natural language understanding with bloom embeddings. 670 convolutional neural networks and incremental parsing, 7(1), (2017).

671 


\section{Supplementary Information}

674 Supplementary Table 1 | Detailed information about our institutional datasets. We gathered

675 examinations for both AP and PA view positions, and automatically generated three labels (positive,

676 negative, ignore) by our natural language processing (NLP) tool which had been developed to convert

677 terms used in radiology reports into the labels. This tool is a rule-based approach that has a dictionary 678 of radiology terms, based on Radex Lexicon [37], and a mapping table of 20 pathological features and 679 the key terms. Label generation of the NLP, using NLTK [38] and Spacy [39], employs two-staged 680 processing for each examination: The first stage extracts radiology terms from a radiology report of 681 the examination using the dictionary while considering both negation and double negation. In the 682 second stage, the NLP determines labels from the terms by a mapping rule defining key terms for 20 683 pathological features. "Ignore" labels are assigned to features when NLP cannot classify the label if 684 there are any conflicting terms in the second stage. The tables show labels' information for the training, 685 validation, and test datasets which were exclusively divided by patients.

\begin{tabular}{|c|c|c|c|c|c|c|c|c|c|c|c|c|c|c|c|c|}
\hline \multirow{4}{*}{$\begin{array}{l}\text { AP view } \\
\text { Fracture }\end{array}$} & \multicolumn{16}{|c|}{ [Gender] Male / Female $=58 \% / 42 \%$, [Age] average $/ \mathrm{std}=62 / 18$} \\
\hline & \multicolumn{6}{|c|}{ Train set } & \multicolumn{6}{|c|}{ Validation set } & \multicolumn{4}{|c|}{ Test set } \\
\hline & \multicolumn{2}{|c|}{ Positive } & \multicolumn{2}{|c|}{ Ignore } & \multicolumn{2}{|c|}{ Negative } & \multicolumn{2}{|c|}{ Positive } & \multicolumn{2}{|c|}{ Ignore } & \multicolumn{2}{|c|}{ Negative } & \multicolumn{2}{|c|}{ Positive } & \multicolumn{2}{|c|}{ Negative } \\
\hline & 3,446 & $4.8 \%$ & 521 & $0.7 \%$ & 68,171 & $94.5 \%$ & 576 & $4.1 \%$ & 89 & $0.6 \%$ & 13,364 & $95.3 \%$ & 12 & $1.2 \%$ & 988 & $98.8 \%$ \\
\hline Non-fracture & 1,275 & $1.8 \%$ & 26 & $0.0 \%$ & 70,837 & $98.2 \%$ & 177 & $1.3 \%$ & 6 & $0.0 \%$ & 13,846 & $98.7 \%$ & 5 & $0.5 \%$ & 995 & $99.5 \%$ \\
\hline Diaphragm & 3,293 & $4.6 \%$ & 116 & $0.2 \%$ & 68,729 & $95.3 \%$ & 580 & $4.1 \%$ & 30 & $0.2 \%$ & 13,419 & $95.7 \%$ & 8 & $0.8 \%$ & 992 & $99.2 \%$ \\
\hline Foreign & 44,034 & $61.0 \%$ & 487 & $4 \%$ & 25,617 & $35.5 \%$ & 9,866 & $70.3 \%$ & 440 & $3.1 \%$ & 3,723 & $26.5 \%$ & 677 & $57.7 \%$ & 23 & $32.3 \%$ \\
\hline Aorta & 4,638 & $6.4 \%$ & 351 & $0.5 \%$ & 67,149 & $93.1 \%$ & 716 & $5.1 \%$ & 51 & $0.4 \%$ & 13,262 & $94.5 \%$ & 63 & $6.3 \%$ & 937 & $93.7 \%$ \\
\hline Cardiomegaly & 10,583 & $14.7 \%$ & 1,904 & $2.6 \%$ & 59,651 & $82.7 \%$ & 2,171 & $15.5 \%$ & 407 & $2.9 \%$ & 11,451 & $81.6 \%$ & 227 & $22.7 \%$ & 773 & $77.3 \%$ \\
\hline ar & 66 & $4 \%$ & & $3 \%$ & 71 & $99.3 \%$ & 9 & $0.3 \%$ & 29 & $0.2 \%$ & 13,961 & $99.5 \%$ & 2 & $0.2 \%$ & 998 & $99.8 \%$ \\
\hline Mediastinum & 1,423 & $2.0 \%$ & 449 & $0.6 \%$ & 70,266 & $97.4 \%$ & 255 & $1.8 \%$ & 88 & $0.6 \%$ & 13,686 & $97.6 \%$ & 16 & $1.6 \%$ & 984 & $98.4 \%$ \\
\hline Cavity/Cyst & 325 & $0.5 \%$ & 110 & $0.2 \%$ & 71,703 & $99.4 \%$ & 116 & $0.8 \%$ & 18 & $0.1 \%$ & 13,895 & $99.0 \%$ & 6 & $0.6 \%$ & 994 & $99.4 \%$ \\
\hline Emphysen & 32 & $1.4 \%$ & 2,261 & $3.1 \%$ & 68,895 & $95.5 \%$ & 170 & $1.2 \%$ & 480 & $3.4 \%$ & 13,379 & $95.4 \%$ & 2 & $0.2 \%$ & 998 & $99.8 \%$ \\
\hline Atelectasis & 36,785 & $51.0 \%$ & 407 & $0.6 \%$ & 34,946 & $48.4 \%$ & 7,734 & $55.1 \%$ & 126 & $0.9 \%$ & 6,169 & $44.0 \%$ & 554 & $55.4 \%$ & 446 & $44.6 \%$ \\
\hline Nodule/mass & 3,663 & $5.1 \%$ & 388 & $0.5 \%$ & 68,087 & $94.4 \%$ & 575 & $4.1 \%$ & 49 & $0.3 \%$ & 13,405 & $95.6 \%$ & 18 & $1.8 \%$ & 982 & $98.2 \%$ \\
\hline Other inters & 2,334 & $3.2 \%$ & 02 & $0.1 \%$ & 69,702 & $96.6 \%$ & 452 & $3.2 \%$ & 20 & $0.1 \%$ & 13,557 & $96.6 \%$ & 8 & $0.8 \%$ & 992 & $99.2 \%$ \\
\hline Pulmonary edema & 18,620 & $25.8 \%$ & 2,921 & $4.0 \%$ & 50,597 & $70.1 \%$ & 4,568 & $32.6 \%$ & 504 & $3.6 \%$ & 8,957 & $63.8 \%$ & 276 & $27.6 \%$ & 724 & $72.4 \%$ \\
\hline pneumonia & 19,581 & $27.1 \%$ & 3,209 & $4.4 \%$ & 49,348 & $68.4 \%$ & 4,322 & $30.8 \%$ & 579 & $4.1 \%$ & 9,128 & $65.1 \%$ & 174 & $17.4 \%$ & 826 & $82.6 \%$ \\
\hline Decreased lung volume & 17,539 & $24.3 \%$ & 879 & $1.2 \%$ & 53,720 & $74.5 \%$ & 3,352 & $23.9 \%$ & 187 & $1.3 \%$ & 10,490 & $74.8 \%$ & 174 & $17.4 \%$ & 826 & $82.6 \%$ \\
\hline Increased lung volume & 820 & $1.1 \%$ & 16 & $0.0 \%$ & 71,302 & $98.8 \%$ & 158 & $1.1 \%$ & 4 & $0.0 \%$ & 13,867 & $98.8 \%$ & 0 & $0.0 \%$ & 1,000 & $100.0 \%$ \\
\hline Other pleural lesions & 1,678 & $2.3 \%$ & 1,942 & $2.7 \%$ & 68,518 & $95.0 \%$ & 329 & $2.3 \%$ & 348 & $2.5 \%$ & 13,352 & $95.2 \%$ & 4 & $0.4 \%$ & 996 & $99.6 \%$ \\
\hline Pleural effusion & 23,020 & $31.9 \%$ & 263 & $0.4 \%$ & 48,855 & $67.7 \%$ & 5,759 & $41.1 \%$ & 51 & $0.4 \%$ & 8,219 & $58.6 \%$ & 348 & $34.8 \%$ & 652 & $65.2 \%$ \\
\hline Pneumothorax & 5,485 & $7.6 \%$ & 1,612 & $2.2 \%$ & 65,041 & $90.2 \%$ & 1,361 & $9.7 \%$ & 351 & $2.5 \%$ & 12,317 & $87.8 \%$ & 71 & $7.1 \%$ & 929 & $92.9 \%$ \\
\hline
\end{tabular}

\begin{tabular}{|c|c|c|c|c|c|c|c|c|c|c|c|c|c|c|c|c|}
\hline \multirow{4}{*}{$\begin{array}{l}\text { PA view } \\
\text { Fracture }\end{array}$} & \multicolumn{16}{|c|}{ [Gender] Male / Female $=50 \% / 50 \%,[$ Age] average $/$ std $=57 / 19$} \\
\hline & \multicolumn{6}{|c|}{ Train set } & \multicolumn{6}{|c|}{ Validation set } & \multicolumn{4}{|c|}{ Test set } \\
\hline & \multicolumn{2}{|c|}{ Positive } & \multicolumn{2}{|c|}{ Ignore } & \multicolumn{2}{|c|}{ Negative } & \multicolumn{2}{|c|}{ Positive } & \multicolumn{2}{|c|}{ Ignore } & \multicolumn{2}{|c|}{ Negative } & \multicolumn{2}{|c|}{ Positive } & \multicolumn{2}{|c|}{ Negative } \\
\hline & 1,919 & $2.9 \%$ & 474 & $0.7 \%$ & 64,155 & $96.4 \%$ & 372 & $3.0 \%$ & 97 & $0.8 \%$ & 11,785 & $96.2 \%$ & 31 & $3.1 \%$ & 969 & $96.9 \%$ \\
\hline Non-fracture & 2,787 & $4.2 \%$ & 25 & $0.0 \%$ & 63,736 & $95.8 \%$ & 524 & $4.3 \%$ & I & $0.1 \%$ & 11,723 & $95.7 \%$ & 0 & $.0 \%$ & 990 & $99.0 \%$ \\
\hline Diaphragm & 2,171 & $3.3 \%$ & 132 & $0.2 \%$ & 64,245 & $96.5 \%$ & 393 & $3.2 \%$ & 19 & $0.2 \%$ & 11,842 & $96.6 \%$ & 15 & $1.5 \%$ & 985 & $98.5 \%$ \\
\hline Foreign body & 16,011 & $24.1 \%$ & 1,189 & $1.8 \%$ & 49,348 & $74.2 \%$ & 3,208 & $26.2 \%$ & 191 & $1.6 \%$ & 8,855 & $72.3 \%$ & 244 & $24.4 \%$ & 756 & $75.6 \%$ \\
\hline Aorta & 272 & $9 \%$ & 03 & $0.6 \%$ & 62,873 & $94.5 \%$ & 509 & $.0 \%$ & 85 & $0.7 \%$ & 11,560 & $94.3 \%$ & 167 & $6.7 \%$ & 833 & $83.3 \%$ \\
\hline Cardiomegaly & 4,289 & $6.4 \%$ & 1,001 & $1.5 \%$ & 61,258 & $92.1 \%$ & 805 & $6.6 \%$ & 170 & $1.4 \%$ & 11,279 & $92.0 \%$ & 104 & $10.4 \%$ & 896 & $89.6 \%$ \\
\hline Hilar area & 97 & $0.3 \%$ & 97 & $0.1 \%$ & 66,254 & $99.6 \%$ & 37 & $0.3 \%$ & 18 & $0.1 \%$ & 12,199 & $99.6 \%$ & 11 & $1.1 \%$ & 989 & $98.9 \%$ \\
\hline Medias & 94 & $1.2 \%$ & 388 & $0.6 \%$ & 65,366 & $98.2 \%$ & 121 & $1.0 \%$ & 87 & $0.7 \%$ & 12,046 & $98.3 \%$ & 8 & $0.8 \%$ & 992 & $99.2 \%$ \\
\hline Cavity/Cyst & 221 & $0.3 \%$ & 78 & $0.1 \%$ & 66,249 & $99.6 \%$ & 38 & $0.3 \%$ & 19 & $0.2 \%$ & 12,197 & $99.5 \%$ & 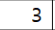 & $0.3 \%$ & 997 & $99.7 \%$ \\
\hline Emphysema & 830 & $1.2 \%$ & 337 & $0.5 \%$ & 65,381 & $98.2 \%$ & 159 & $1.3 \%$ & 61 & $0.5 \%$ & 12,034 & $98.2 \%$ & 2 & $0.2 \%$ & 998 & $99.8 \%$ \\
\hline Atelectasis & 12,905 & $19.4 \%$ & 245 & $0.4 \%$ & 53,398 & $80.2 \%$ & 2,539 & $20.7 \%$ & 40 & $0.3 \%$ & 9,675 & $79.0 \%$ & 235 & $23.5 \%$ & 765 & $76.5 \%$ \\
\hline Nodule/mass & 3,981 & $6.0 \%$ & 503 & $0.8 \%$ & 62,064 & $93.3 \%$ & 733 & $6.0 \%$ & 92 & $0.8 \%$ & 11,429 & $93.3 \%$ & 36 & $3.6 \%$ & 964 & $96.4 \%$ \\
\hline Other interstitial opacity & 4,047 & $6.1 \%$ & 209 & $0.3 \%$ & 62,292 & $93.6 \%$ & 780 & $6.4 \%$ & 37 & $0.3 \%$ & 11,437 & $93.3 \%$ & 12 & $1.2 \%$ & 988 & $98.8 \%$ \\
\hline Pulmonary edema & 2,417 & $3.6 \%$ & 4,121 & $6.2 \%$ & 60,010 & $90.2 \%$ & 492 & $4.0 \%$ & 783 & $6.4 \%$ & 10,979 & $89.6 \%$ & 35 & $3.5 \%$ & 965 & $96.5 \%$ \\
\hline pneumonia & 8,602 & $12.9 \%$ & 4,836 & $7.3 \%$ & 53,110 & $79.8 \%$ & 1,663 & $13.6 \%$ & 939 & $7.7 \%$ & 9,652 & $78.8 \%$ & 123 & $12.3 \%$ & 877 & $87.7 \%$ \\
\hline Decreased lung volume & 4,530 & $6.8 \%$ & 274 & $0.4 \%$ & 61,744 & $92.8 \%$ & 833 & $6.8 \%$ & 50 & $0.4 \%$ & 11,371 & $92.8 \%$ & 23 & $2.3 \%$ & 977 & $97.7 \%$ \\
\hline Increased lung volume & 2,290 & $3.4 \%$ & 13 & $0.0 \%$ & 64,245 & $96.5 \%$ & 422 & $3.4 \%$ & 4 & $0.0 \%$ & 11,828 & $96.5 \%$ & 8 & $0.8 \%$ & 992 & $99.2 \%$ \\
\hline Other pleural lesions & 1,659 & $2.5 \%$ & 1,372 & $2.1 \%$ & 63,517 & $95.4 \%$ & 370 & $3.0 \%$ & 261 & $2.1 \%$ & 11,623 & $94.9 \%$ & 8 & $0.8 \%$ & 992 & $99.2 \%$ \\
\hline Pleural effusion & 7,044 & $10.6 \%$ & 299 & $0.4 \%$ & 59,205 & $89.0 \%$ & 1,512 & $12.3 \%$ & 57 & $0.5 \%$ & 10,685 & $87.2 \%$ & 136 & $13.6 \%$ & 864 & $86.4 \%$ \\
\hline Pneumothorax & 885 & $1.3 \%$ & 388 & $0.6 \%$ & 65,275 & $98.1 \%$ & 155 & $1.3 \%$ & 68 & $0.6 \%$ & 12,031 & $98.2 \%$ & 9 & $0.9 \%$ & 991 & 99.19 \\
\hline
\end{tabular}


Supplementary Table 2 | Model performance. xAl model performance for 20 features was evaluated on PA test datasets of our institution (PA view in Supplementary Table 1) based on both NLP and human labels. The $95 \% \mathrm{Cls}$ on the metrics were provided in parentheses (Statistical analyses in Methods). Out of 20 features, in this study, we selected 5 typical features that were commonly defined in three public datasets.

\begin{tabular}{|c|c|c|c|c|c|}
\hline Class & Test (NLP) & Test (Human) & Class & Test (NLP) & Test (Human) \\
\hline Fracture & $0.77(0.67,0.86)$ & $0.85(0.77,0.91)$ & Nodule/mass & $0.68(0.60,0.76)$ & $0.80(0.71,0.88)$ \\
\hline Non-fracture & $0.91(0.85,0.96)$ & $0.99(0.98,1.00)$ & Other interstitial opacity & $0.83(0.78,0.87)$ & $0.94(0.89,0.98)$ \\
\hline Diaphragm & $0.93(0.90,0.96)$ & $0.97(0.94,0.99)$ & Pulmonary edema & $0.95(0.91,0.97)$ & $0.98(0.97,0.99)$ \\
\hline Foreign body & $0.92(0.90,0.94)$ & $0.95(0.93,0.97)$ & pneumonia & $0.77(0.73,0.82)$ & $0.90(0.88,0.93)$ \\
\hline Aorta & $0.91(0.88,0.94)$ & $0.94(0.92,0.96)$ & Decreased lung volume & $0.92(0.89,0.94)$ & $0.98(0.96,0.99)$ \\
\hline Cardiomegaly & $0.93(0.91,0.95)$ & $0.97(0.95,0.98)$ & Increased lung volume & $0.91(0.86,0.95)$ & $0.98(0.96,0.99)$ \\
\hline Hilar area & $0.82(0.58,0.98)$ & $0.94(0.90,0.98)$ & Other pleural lesions & $0.85(0.76,0.92)$ & $0.98(0.94,1.00)$ \\
\hline Mediastinum & $0.92(0.86,0.97)$ & $0.98(0.95,1.00)$ & Pleural effusion & $0.97(0.96,0.98)$ & $0.98(0.97,0.99)$ \\
\hline Cavity/Cyst & $0.87(0.76,0.95)$ & $0.94(0.87,1.00)$ & Pneumothorax & $0.89(0.78,0.97)$ & $0.95(0.91,0.98)$ \\
\hline Emphysema & $0.94(0.90,0.98)$ & $1.00(0.99,1.000)$ & \multirow{2}{*}{ Mean AUROC } & \multirow{2}{*}{$\mathbf{0 . 8 8}(\mathbf{0 . 8 6 , 0 . 8 9 )}$} & \multirow{2}{*}{$\mathbf{0 . 9 5}(\mathbf{0 . 9 4}, \mathbf{0 . 9 6})$} \\
\cline { 1 - 2 } & \multicolumn{2}{|c|}{$0.94(0.93,0.96)$} & & &
\end{tabular}
selection algorithm determines both a label (positive, negative, or unlabeled) and a mode (selfannotation or re-annotation) for each test image by using uses pSim and a selected pSim threshold values, where $T H_{\text {pos }}$ was set to 0.5 in this study.

\section{Mode selection algorithm}

Input: predicted probability for c-class $\left(y^{c}\right)$, Confidence $_{P}$, Confidence $_{N}$, and patch similarity

$\%\left[\right.$ step-1] To divide into two groups by $y^{c}$ and $T H_{\text {pos }}$ : positive or negative candidates If $y^{c} \geq T H_{\text {pos }}$ : then

$\%[$ step-2] To decide mode and annotation for the positive candidates

$\%$ Probability of Similarity, $p$ Sim

$\mathrm{pSim}=2 \cdot$ Confidence $e_{P} \cdot p$ Similarity $/\left(\right.$ Confidence $_{P}+$ pSimilarity $)$

If $p$ Sim $>=p$ Sim threshold value ( $P P V, N P V=1)$ : then

Mode $=$ Self-annotation mode

Label $=1 \%$ Positive label

Else

Mode $=$ Re-annotation mode

Label $=-1 \%$ unlabeled

Else

$\%[$ step-2] To decide mode and annotation for the negative candidates

pSim $=$ Confidence ${ }_{N}$

If $p$ Sim $>=p$ Sim threshold value (PPV, $N P V=1)$ : then

Mode $=$ Self-annotation mode

Else

Label $=0 \%$ Negative label

Mode $=$ Re-annotation mode

Annotation $=-1 \%$ unlabeled 


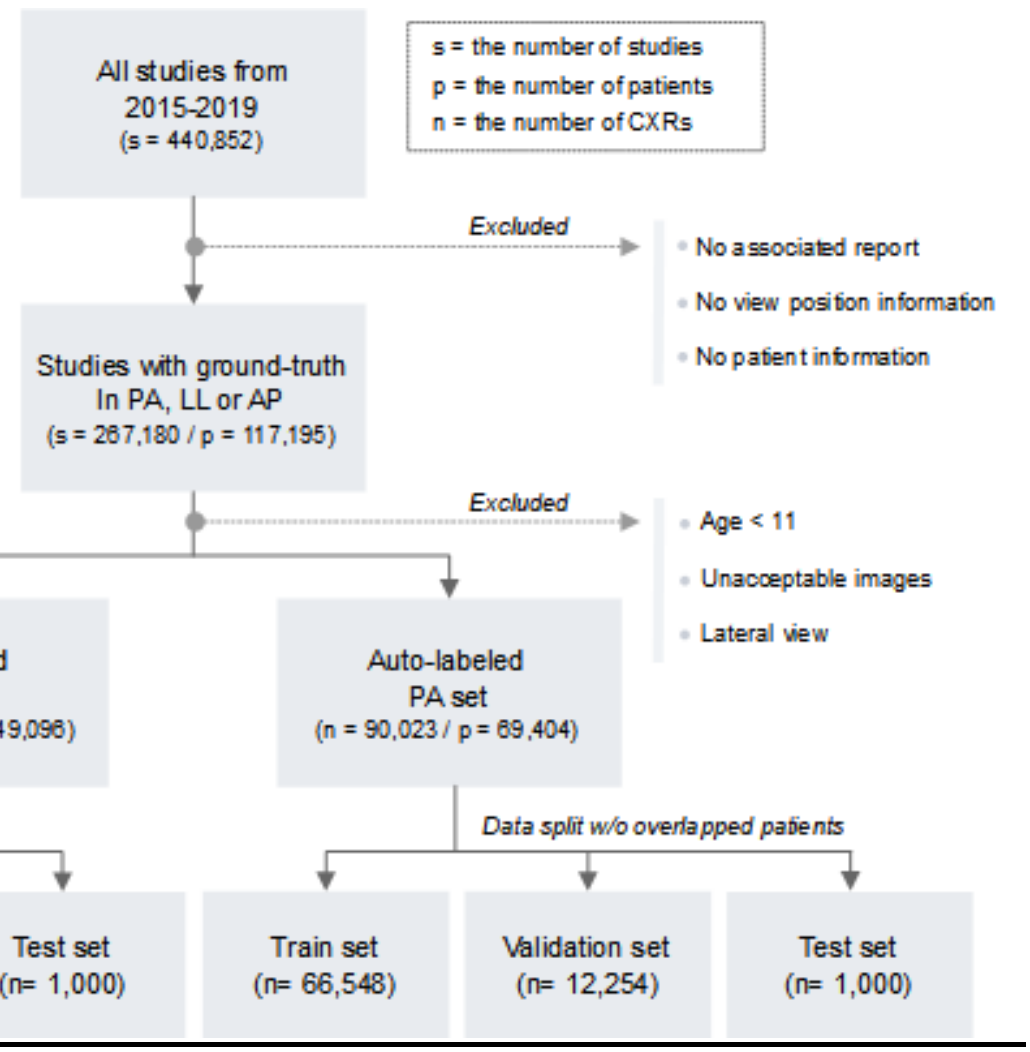

702 Supplementary Figure 1 | CXR data collection for the XAI model development. Each dataset 703 for AP and PA view positions collected and mapped one-on-one to the annotations extracted by 704 our NLP tool from the corresponding radiological reports. For each view position, training, 705 validation, and test datasets were divided without overlapped patients or duplicated cases. 